\title{
The orientation of Titan's dayside ionosphere and its effects on Titan's plasma interaction
}

\author{
Stephen A. Ledvina ${ }^{1}$, Stephen H. Brecht ${ }^{2}$, and Thomas E. Cravens ${ }^{3}$ \\ ${ }^{1}$ Space Sciences Laboratory, UC Berkeley, 7 Gauss Way, Berkeley, CA, USA \\ ${ }^{2}$ Bay Area Research Corp. P.O. Box 366, Orinda, CA 94563, USA \\ ${ }^{3}$ Department of Physics and Astronomy, University of Kansas, Lawrence, KS, USA
}

(Received March 16, 2011; Revised July 29, 2011; Accepted August 3, 2011; Online published March 8, 2012)

\begin{abstract}
A hybrid particle code has been used to examine how Titan's interaction with Saturn's magnetosphere is effected by the orientation of the dayside ionosphere with respect to the incident magnetospheric flow. The hybrid code self-consistently includes a version of Titan's ionosphere represented by 7 generic ion species, over 40 ionneutral chemical reactions, ion-neutral collisions and Hall and Pederson conductivities. Emphasis is placed on what effects the orientation angle has on the ion loss rates, ion densities, and the electric and magnetic fields. The results are analyzed and regardless of the orientation angle the ionosphere is found to be within photochemical equilibrium below $1200 \mathrm{~km}$ altitude. The ion loss rates and field structures also show little dependence on the orientation of the dayside ionosphere. It is found to first order illumination angle does not have a significant effect on these features of the Titan interaction.
\end{abstract}

Key words: Titan, ionosphere, plasma interaction with Saturn's magnetosphere.

\section{Introduction}

Understanding the dynamics of Titan's plasma interaction and how its ionosphere couples to Saturn's magnetosphere is a complex problem. Full 3-dimensional simulations that include accurate descriptions of the plasma, the ionospheric chemistry and the neutral atmosphere are a critical tool to help solve the problem. This paper presents results from 3-d hybrid (kinetic ions, fluid electrons) simulations of Titan's plasma interaction that include ionospheric chemistry, ion-neutral and electron-neutral interactions.

The first in-situ observations of Titan's interaction region were made by a single flyby of Voyager 1 through Titan's wake in 1980. No intrinsic magnetic field was found at Ti$\tan$ (Ness et al., 1982). Titan was found to have an extensive ionosphere that interacts directly with Saturn's magnetospheric plasma and fields (Hartle et al., 1982; Bird et al., 1997). The interaction leads to Saturn's magnetic field piling up and draping around Titan forming an induced magnetosphere (Ness et al., 1982). Voyager also observed Titan's ionospheric ions being lost to Saturn's magnetosphere (Hartle et al., 1982). Net ion loss rates were estimated to be 1-3 × 10 24 ions/s (Eviatar et al., 1982; Gurnett et al., 1982).

After the first Titan encounter it was speculated that Titan's interaction with Saturn's magnetosphere could be more complex than the Voyager picture. Unlike the solar wind's interaction with the planets, Titan's interaction with Saturn's magnetospheric flow does not have to coincide with the solar produced ionosphere. While the plan-

Copyright (C) The Society of Geomagnetism and Earth, Planetary and Space Sciences (SGEPSS); The Seismological Society of Japan; The Volcanological Society of Japan; The Geodetic Society of Japan; The Japanese Society for Planetary Sciences; TERRAPUB.

doi:10.5047/eps.2011.08.009 ets only interact with the solar wind, Titan can be found in Saturn's magnetosheath, tail, or in the flanks of the planet. These different orientations lead to different plasma conditions at Titan (cf. Wolf and Neubauer, 1982; Cravens et al., 1998; Ledvina and Cravens, 1998; Ledvina et al., 2004a; Bertucci et al., 2009; Rymer et al., 2009).

Cassini spacecraft made its first flybys of Titan in 2004. Since then Cassini has made over 76 flybys of Titan. Cassini has confirmed many of the previous Voyager results such as the lack on an intrinsic magnetic field and an induced magnetosphere (Backes et al., 2005). However, Cassini has also found the Titan interaction to be even more variable and complex than previously thought. The ionosphere contained several species with mass greater than $100 \mathrm{amu}$ (Waite et al., 2005) as well as negative ions (Coates et al., 2007). A substantial night side ionosphere was found (Wahlund et al., 2005; Cravens et al., 2006; Ågren et al., 2007, 2009). There has been some debate over the source of the night side ionosphere. The short lived ions in the night side ionosphere may be created by super thermal electron impact ionization (Gan et al., 1992; Ågren et al., 2007; Cravens et al., 2009), while plasma transport from the day side to the night side of the long lived ion species may also occur (Cui et al., 2009).

Cassini made the first measurements of Titan's ionospheric conductivities (Rosenqvist et al., 2009). The peak Hall and Pederson conductivities were found to be dependent on the solar illumination and the magnetospheric conditions. The conductivities were typically higher on the sunlit side but depended strongly on the magnetic field magnitude and the field draping configuration. A consistent double peak was found in the altitude profile of the Pederson conductivity. The first peak was within $1300-1400 \mathrm{~km}$ al- 
titude. The second peak was located below $1000 \mathrm{~km}$, were the magnetic field strength drops sharply but the electron density is still high. The Pedersen conductance was always found to be higher than the local Alfvén conductance (from a factor of 4 to 100 times greater). The Pederson conductivity profile should give rise to complicated ionosphericatmospheric dynamics. It also implies that the conditions for the ionosphere to reflect or absorb Alfven waves are highly variable and strongly dependent on the magnetic field strength (Rosenqvist et al., 2009).

Recently Ågren et al. (2011) combined data from radio and plasma wave science, the magnetometer, the plasma spectrometer with the ionospheric conductivities. They found currents flowing in Titan's ionosphere ranging from $10-100 \mathrm{nA} / \mathrm{m}^{2}$. Below altitudes of $1400 \mathrm{~km}$ (the exobase) the currents were principally field aligned (Hall), the Pederson currents were found to be negligible. Perpendicular electric fields ranging from $0.5-3 \mu \mathrm{V} / \mathrm{m}$ were associated with the observed currents.

The plasma and field conditions along Titan's orbit have been found by Cassini to be more variable than previously thought (cf. Bertucci et al., 2009). This variability was found to be crucial to understanding aspects of the T9 observations by Modolo et al. (2007). After the Voyager encounter the magnetic field was thought to be nearly perpendicular to Titan's orbital plane throughout the orbit. Cassini has found this to be the exception rather than the rule. Arridge et al. (2007) has interpreted Cassini magnetometer observations as a thin current sheet in the form of a magnetodisc. A follow up study by Arridge et al. (2008) found that the magnetodisc, and hence Saturn's magnetosphere, is strongly influenced by solar wind dynamic pressure. The magnetodisc is warped by the solar wind. This warping exposes Titan to quasi-dipolar fields in the noon sector and planet-ward, swept-back fields in the dawn, dusk and midnight sectors. Depending on where Titan is in relationship to the magnetodisc it is exposed to either: (1) a "magnetodisc lobe' regime where the plasma beta is low and fields are radially 'stretched' and usually stronger or (2) a 'current sheet' regime characterized by quasi-dipolar, relatively weak fields and a high-beta plasma (Bertucci et al., 2009).

Additional studies of electron density in the magnetodisc have found that periodicity in the magnetosphere are due to plasma outflows driven by centrifugal force. This material originates from Enceladus and works its way out to Titan. This periodicity can cause a larger variability at Titan than the solar wind (cf. Persoon et al., 2005, 2006; Morooka et al., 2009).

Simon et al. (2010a) classified the first 62 Titan flybys as lobe-type or current sheet. They concluded that not a single flyby up to then matched the Voyager picture of a homogeneous background magnetic field perpendicular to the moon's orbital plane. The magnetic field varied on time scales ranging from a few minutes to several hours. These observations were later verified in a follow up study using data collected along Titan's orbit when Titan was not present (Simon et al., 2010b). To further complicate things there are times when Titan has been observed within Saturn's magnetosheath (cf. Bertucci et al., 2008; Garnier et $a l ., 2009$ ). Understanding the magnetospheric variability is crucial to understanding Titan's interaction. Asymmetries in the observed ion densities and composition downstream of Titan during the T9 flyby were attributed by Modolo et al. (2007) as being due to the variability in the magnetic field morphology.

Interpretations of the Voyager PLS data found a two component plasma consisting of $\mathrm{H}^{+}$and $\mathrm{N}^{+}$with number densities of 0.1 and $0.2 \mathrm{~cm}^{-3}$ respectively. Both species were found to have gyroradii comparable to Titan $\left(1 R_{\mathrm{T}}=\right.$ $2575 \mathrm{~km}$ ). Each species was fitted with a Maxwellian distribution function with a thermal speed of $200 \mathrm{~km} / \mathrm{s}$ (Hartle et al., 1982). It was suggested by Eviatar et al. (1982) that the $\mathrm{N}^{+}$originated from Titan and formed a torus about its orbit. Based on the assumption that the $\mathrm{N}^{+}$component could be pickup ions, Ledvina et al. (2005) showed that the PLS data could be fit by a shell distribution. The post Cassini picture has found that the $\mathrm{N}^{+}$component was really $\mathrm{O}^{+}$and is thought to originate from the icy satellites and moons (Szego et al., 2005). Szego et al. (2005) fit the incident $\mathrm{O}^{+}$to a shell distribution function. The Cassini plasma data along Titan's orbit have been fit to Maxwellian, shell and kappa distribution functions. The exact distribution function of the incident plasma is still an issue that remains to be settled.

Simulations have been used over the years to understand Titan's interaction with Saturn's magnetosphere. Initial 1D simulations included single and multifluid MHD and hydrodynamics were used to predict the magnetic flux into the ionosphere and ion loss out the tail (e.g., Ip, 1990; Keller and Cravens, 1994; Keller et al., 1994). These simple 1-D simulations were followed by a multispecies 2-D MHD model (Cravens et al., 1998) and a single fluid MHD model (Ledvina and Cravens, 1998). These early multidimensional simulations confirmed the basic post-Voyager picture of Titan's interaction but they did not have a realistic ionosphere. Higher resolution MHD studies followed (Kabin et al., 1999; Kopp and Ip, 2001; Nagy et al., 2001; Ma et al., 2004, 2006, 2007; Backes et al., 2005) as did a multifluid simulation by Snowden et al. (2007). The later studies by Ma et al., were multispecies ( 7 continuity equations) but single fluid (1 momentum equation), they included ion-neutral collisions and chemistry. Their latest results Ma et al. (2007) also include the Hall term in the MHD equations.

These simulations have been successful at describing many aspects of Titan's plasma interaction. However, they are all based on the fluid approximation. Gyroradius effects and none isotropic pressures are not described in this approximation. The fluid approximation cannot provide a complete description of the interaction because of the size of the ion gyroradii near Titan. This issue was recognized early on with initial studies of the kinetic effects being carried out by particle tracing in ideal and MHD fields (cf. Luhmann, 1996; Ledvina et al., 2000, 2004b, 2005; Tseng et al., 2008). Particle tracing gives a better sense of the importance of gyroradius effects than MHD simulations but it is not self-consistent. There is no feedback between the particle motion and the fields. Self-consistent hybrid simulations (kinetic ions, fluid electrons) have been carried to account for the ion gyroradii effects near Titan (e.g. Brecht 


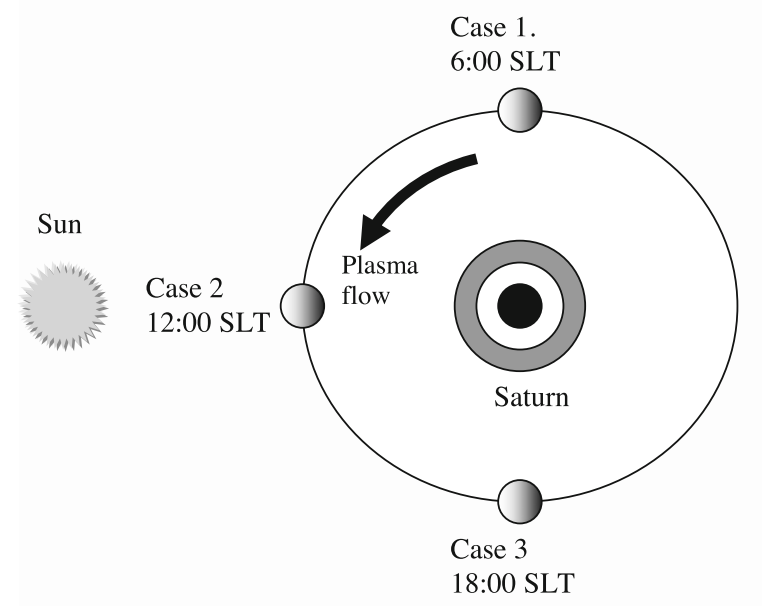

Fig. 1. This is a schematic looking down on Saturn, showing the location of Titan along its orbit for each simulation case.

et al., 2000; Ledvina et al., 2004a; Sillanpää et al., 2006; Simon et al., 2006; Modolo et al., 2007; Müller et al., 2010). The major limitation to the hybrid simulation performed to date has been their lack of any description of the ionosphere.

The research reported here addresses this limitation in the previous hybrid simulations. We have applied our experience from incorporating ionospheric processes into hybrid simulations of Mars (cf. Brecht and Ledvina, 2012) to Titan. The resulting ionosphere contains seven generic ion species and the same ion-neutral chemistry used by Ma et al. in their Titan simulations. There are eleven neutral species whose altitude profiles are based on Cassini observations and the model of Toublanc et al. (1995). The ions not only interact with the neutrals chemically but also through collisions via their respective collisional cross sections. The ion-neutral collision process is also used to self-consistently include the Hall and Pederson conductivities into the electric field calculation. Photoionization on the dayside is the major ionization source, but secondary electron impact ionization is also included. The night side ionosphere is created from superthermal electron impact ionization (cf. Gan et al., 1992; Galand et al., 1999; Ågren et al., 2007; Cravens et al., 2009). The effect of Titan's gravity on the ion motion is also included.

As previously stated there are many variables to Titan's interaction with Saturn's magnetosphere. This paper focuses on only one the angle of the solar illumination and what effects it has on ion escape, plasma density and electromagnetic field structures around Titan. We examine three cases shown in Fig. 1. The first represents Titan at 06:00 Saturn local time. When Titan is at this location the wake side hemisphere is illuminated. The second case occurs when Titan is at 12:00 Saturn local time. Here the antiSaturn facing hemisphere is illuminated. The final case has the ram side hemisphere illuminated. This condition occurs when Titan is at 18:00 Saturn local time. No doubt the magnetospheric conditions are different at each of these locations. Different magnetospheric conditions will lead to different ion loss rates. Including the differences in the magnetospheric conditions in the simulations could obscure any effect the orientation with respect to the flow of the dayside ionosphere has on Titan's plasma interaction. Therefore the incident plasma and field conditions are held constant in each case to isolate only the effects due to orientation of the dayside ionosphere. The magnetic field is taken to be $5 \mathrm{nT}$ pointing southward perpendicular to Titan's orbital plane. We take the incident plasma to consist of only $\mathrm{O}^{+}$with a density of $0.2 \mathrm{~cm}^{-3}$ moving with a speed of $120 \mathrm{~km} / \mathrm{s}$ with respect to Titan. The incident electrons have a temperature of $200 \mathrm{eV}$. We do not address the issue of the upstream ion distribution function but adopt the simplest approach and load the ions with a beam distribution.

Sillanpää et al. (2006) have previously examined the effect of the angle of solar illumination with respect to the incident flow. They concluded that the illumination angle did not make much of a difference with regard to the amount of plasma lost from Titan. Their results were cutting edge at the time. However, several simplifying assumptions were necessary so that the simulations could be performed. The inner boundary of their simulation was Titan's exobase. This surface was modeled as a perfectly conducting sphere of radius $4175 \mathrm{~km}$. They were only able to include two ion species. The first, $\mathrm{N}_{2}{ }^{+}$was emitted uniformly from Titan's exobase. The second, $\mathrm{CH}_{4}{ }^{+}$was photoionized from a Chamberlain density function. They did not include any ionospheric processes in their simulations. Furthermore their simulations were limited to less than 2 million particles to represent both the magnetospheric and ionospheric plasmas. Given the complexity of Titan's ionosphere and its importance as a boundary condition it is worth re-examining the effects of the illumination angle on ion loss from Titan.

The next section provides a discussion of the models used in the simulations. A discussion of the ion loss for each case follows. The last two sections will provide a discussion of the results and some conclusions.

\section{Simulation Models \\ 2.1 The plasma model}

The plasma is modeled using the hybrid approximation. The plasma is assumed to be charge-neutral. The ions are 
treated as kinetic particles, while the electrons are treated as a massless fluid. The displacement current is dropped from Maxwell's equations by use of the Darwin approximation (cf. Brecht and Ledvina, 2006). This results in the removal of light waves from the simulation. However, a full set of electromagnetic waves up to and including the ion Whistler waves are retained. The quasi-neutrality assumption means that charge separation and electrostatic instabilities are also removed from the simulations. While the massless electron assumption means that high frequency modes due to the electron motion cannot be simulated. The lack of charge separation and $m_{e}$ means that scale sizes need to be much larger than the Debye length, the magnetic skin depth and the electron skin depth. Under these assumptions the resulting set of equations describes the plasma:

Quasi-neutrality

$$
n_{e}=n_{i}
$$

Ampere's Law

$$
\nabla \times \mathbf{B}=(4 \pi / c) \mathbf{J}
$$

Faraday's Law

$$
c \nabla \times \mathbf{E}=-\partial \mathbf{B} / \partial t
$$

The ion equations of motion

$$
\begin{aligned}
& m_{i} d \mathbf{v}_{i} / d t=q_{i} \mathbf{E}+q_{i} \mathbf{v}_{i} \times \mathbf{B} / c-q_{i} \eta \mathbf{J} \\
& d \mathbf{x}_{i} / d t=\mathbf{v}_{i}
\end{aligned}
$$

The inertialess electron momentum equation

$$
0=-e n_{e} \mathbf{E}+\mathbf{J}_{e} \times \mathbf{B} / c-\nabla p_{e}+e n_{e} \eta \mathbf{J}
$$

The electron temperature equation

$$
\partial T_{e} / \partial t=-\mathbf{u}_{e} \cdot \nabla T_{e}-3 / 2 T_{e} \nabla \cdot \mathbf{u}_{e}+2 / 3 \eta \mathbf{J}^{2} / n_{e}
$$

where $\eta$ is the plasma resistivity (the inverse of the conductivity, $\sigma$ ).

The electric field used in Eqs. (3) and (4) is found from Eq. (6). The electron current in Eq. (6) is found by subtracting the ion current obtained by the particles from the total current found with Eq. (2). The equations are solved using a predictor-corrector algorithm developed by Harned (1982). Further discussion about the hybrid approximation, solution algorithms and applications can be found in Ledvina et al. (2008) and references there in.

When the neutral densities become significant ionneutral and electron neutral collisions will modify the electric field via the Hall and Pederson conductivities. These processes are important in Titan's ionosphere and indeed the conductivities have been measured as mentioned above.

The generalized Ohm's law can be written in vector form as:

$$
\mathbf{J}=\sigma_{\|} \mathbf{E}_{\|}^{\prime}+\sigma_{\perp} \mathbf{E}_{\perp}^{\prime}+\sigma_{H} \mathbf{b} \times \mathbf{E}_{\perp}^{\prime}
$$

where $\mathbf{J}$ is the current density, $\mathbf{E}^{\prime}=\mathbf{E}-\nabla p_{e} / e n_{e}, \mathbf{E}_{\|}^{\prime}$ and $\mathbf{E}_{\perp}^{\prime}$ are the components of $\mathbf{E}^{\prime}$ that are parallel and perpendicular to the magnetic field, $\mathbf{b}=\mathbf{B} /|\mathbf{B}| . \sigma_{\|}$is the conductivity along the magnetic field, $\sigma_{\perp}$ is the conductivity perpendicular to the magnetic field (also called the Pederson conductivity) and $\sigma_{H}$ is the Hall conductivity. This expression can inverted to find $\mathbf{E}^{\prime}$ and then $\mathbf{E}$. The generalized electric field then becomes (Mitchner and Kruger, 1973):

$$
\mathbf{E}=\frac{\mathbf{J}+\beta_{e} \mathbf{J} \times \mathbf{b}+s \mathbf{b} \times(\mathbf{J} \times \mathbf{b})}{\sigma_{\|}}-\frac{1}{e n_{e}} \nabla p_{e}
$$

where $\beta_{e}$ is the electron Hall parameter, $\left(\omega_{e n} / \nu_{e H}\right)$ with $\omega_{e H}=e B / m_{e}$, the electron cyclotron frequency, and $v_{e H}$ is the electron collision frequency (electron-neutral and electron-ion) and the ion slip factor, $s$, is $\left(\left(\rho_{n} / \rho\right)^{2} \beta_{e} \beta_{i}\right)$ where $\rho_{n}$ is the neutral density, $\rho$ is the total density (ion + neutral $), \beta_{i}$ is the ion Hall parameter $\left(\omega_{i n} / v_{i n}\right)$ and $\sigma_{\|}$is given by $n_{e} e^{2} / m_{e} v_{e H}$. In the limit where the neutral density or the collision frequencies go to zero, this equation returns to Eq. (6). The effects due to the Hall and Pederson conductivities are folded into the $s \mathbf{b} \times(\mathbf{J} \times \mathbf{b}) / \sigma_{\|}$term. The electron-ion and electron-neutral collision frequencies were taken from Mitchner and Kruger (1973). The ionneutral collision frequencies were taken from Schunk and Nagy (2000).

The quasi-neutrality condition (Eq. (1)) is the basis of any hybrid or MHD model. Strictly speaking it is not necessary for the electron density to be equal to the ion density only that the density of negative charge carriers equals the density of the positively charged carriers and charge separation not be allowed. At Titan $n_{e}=n_{i}$ is a good approximation above $1200 \mathrm{~km}$. However, below that altitude negatively charged massive ions have been found in Titan's ionosphere (Coates et al., 2007). The mass distribution of the negative ions in Titan's ionosphere was found to increase at low altitudes and at high latitudes. There was also a weak mass dependence on the solar zenith angle Coates et al. (2009). The creation and dynamics of the negative ions is not well understood and is a topic of current research. The simulation results presented here do not include a model for the negative ions.

The simulation are performed in Titan centered Cartesian coordinates extending $\pm 10 R_{\mathrm{T}}$ in each direction. A cell size of $250 \mathrm{~km}$ is used to resolve the electric and magnetic fields. Chemistry and ion-neutral collisions are resolved on different grids and are described in the following subsections. Ions are injected upstream of Titan with a mass of $16 \mathrm{amu}$ and a density of $0.2 \mathrm{~cm}^{-3}$. We take the simplest approach possible and load the upstream ions cold with an $E \times B$ drift speed of $120 \mathrm{~km} / \mathrm{s}$ with respect to Titan. The incident magnetic field is $5 \mathrm{nT}$ and is perpendicular to Titan's orbital plane. Below an altitude of $1400 \mathrm{~km}$ the electron temperature profile is based on the Langmuir probe results (Ågren et al., 2009) and is held constant.

\subsection{The neutral atmosphere model}

The neutral atmosphere used in the simulations is assumed to be stationary, spherically symmetric and consist of 11 neutral species, $\mathrm{H}, \mathrm{H}_{2}, \mathrm{CH}_{4}, \mathrm{HCN}, \mathrm{N}_{2}, \mathrm{C}_{2} \mathrm{H}_{2}, \mathrm{C}_{2} \mathrm{H}_{4}$, $\mathrm{C}_{2} \mathrm{H}_{6}, \mathrm{C}_{3} \mathrm{H}_{4}, \mathrm{C}_{4} \mathrm{H}_{2}$ and $\mathrm{HC}_{3} \mathrm{~N}$. Of these species the density profiles of $\mathrm{CH}_{4}$ and $\mathrm{N}_{2}$ were taken from the INMS profiles during the TA encounter (Waite et al., 2005). The neutral profiles for the other species came from the model of Toublanc (cf. Keller et al., 1998; Toublanc et al., 1995). 


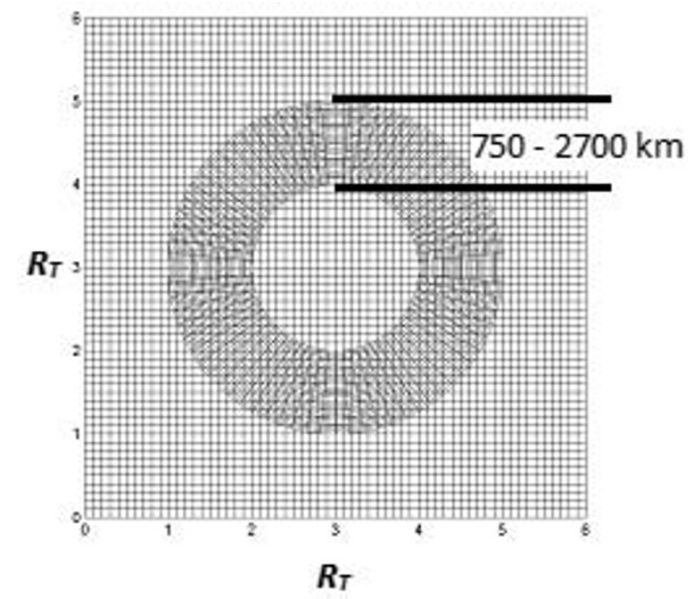

Fig. 2. This is a schematic of the various grids used in the simulations. The electromagnetic fields are solved on the Cartesian grid, while the ion-neutral chemistry and collisions are solved on the spherical grid. The radius of the "spherical" grid ranges between $750 \mathrm{~km}$ and $2700 \mathrm{~km}$.

The neutral collision operators are located on a nonorthogonal spherical grid $(r, \theta, \phi$ see Fig. 2$)$. This grid extends radially from $750-2700 \mathrm{~km}$ in altitude with $\Delta r=50$ $\mathrm{km}$ and $\Delta \theta, \Delta \phi=1.9^{\circ}$. Every particle whose position overlaps this neutral grid is subject to ion-neutral interactions. Each particle collides with each neutral species using their relative velocities and their density and mass dependent collision cross-sections. These ion-neutral collisions result in a frictional drag applied to each particles motion and the Hall and Pederson conductivities in the electric field.

\subsection{The ionosphere}

Titan's ionosphere is the source of new plasma added to Saturn's magnetosphere. It also acts as a plasma sink to the magnetospheric plasma. The extent that these processes occur is controlled by a complex ion-neutral chemical network (cf. Keller et al., 1998; Fox and Yelle, 1997). It is not currently feasible to include such a detailed chemistry networks into three-dimensional simulations. Instead we have included a simplified version of the Keller et al. (1998) ionneutral chemistry network. The reduced model groups several similar species into 7 generic ion species (see Table 1). Photoionization and secondary electron impact ionization rates of the $\mathrm{L}^{+}, \mathrm{M}^{+}$and $\mathrm{H}^{+}$species are pre-computed as a function of altitude and solar zenith angle using a solar minimum flux (cf. Cravens et al., 2005). Electron impact ionization from Maxwellian electrons with $n_{e}=0.1 \mathrm{~cm}^{-3}$ and $T_{e}=100 \mathrm{eV}$ moving along parabolic field lines is used to create a night side ionosphere (Cravens et al., 2009). This is consistent with the electron spectrum measured during the TA encounter. All other species are created via ion-neutral reactions of which there are over 40 (see Appendix A). This is the same chemistry model used by Ma et al. $(2004,2006$, 2007).

The chemical model was run for $20,000 \mathrm{~s}$ to reach equilibrium below $2000 \mathrm{~km}$ altitude, the density profiles for noon and midnight solar conditions are shown in Fig. 3. The $\mathrm{L}^{+}, \mathrm{M}^{+}$and $\mathrm{H}^{+}$are the dominant species above altitudes of $1800 \mathrm{~km}$. The night side ionosphere (Fig. 3(b)) has

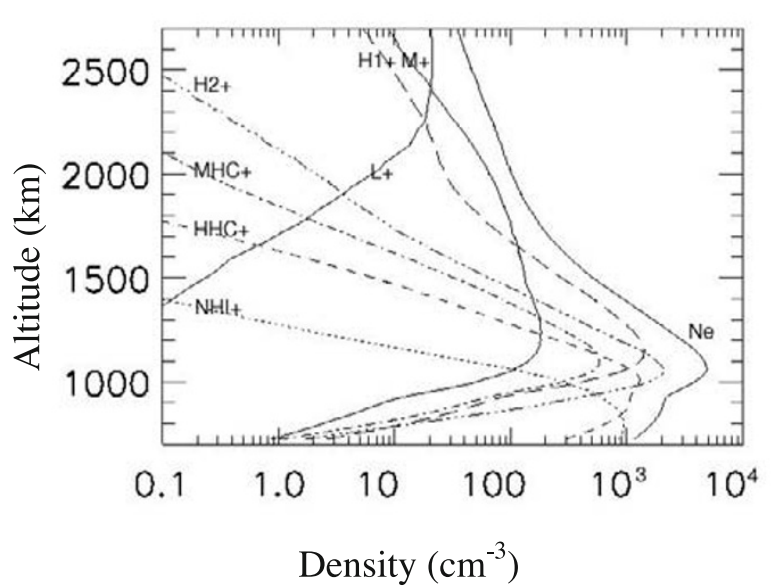

(A)

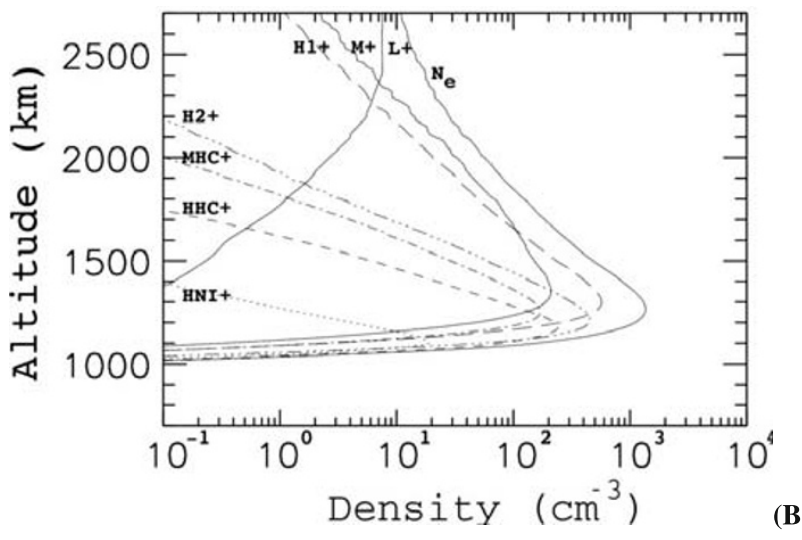

Fig. 3. (A) Ionospheric density profiles versus altitude for the ion-neutral chemical model with a $0^{\circ}$ solar zenith angle (sza, noon Titan time). The day side ionosphere is driven by photoionization. The peak electron density is $5000 \mathrm{~cm}^{-3}$ at $1050 \mathrm{~km}$ altitude. (B) Ion density profiles versus altitude from the ion-neutral chemical model with a $180^{\circ}$ sza (midnight Titan time). The night side ionosphere is driven by super thermal electron impact ionization. The electron density peaks is 1300 $\mathrm{cm}^{-3}$ at $1225 \mathrm{~km}$ altitude. The night side ionosphere drops out below $1100 \mathrm{~km}$.

a peak electron density of about $1300 \mathrm{~cm}^{-3}$, or $25 \%$ of the day side density peak. However the night side density goes to zero below an altitude of $1000 \mathrm{~km}$, while the dayside ionosphere extends down below the simulation grid. Based on the density profiles shown in Fig. 3 the ionosphere will not be symmetric about Titan. The day side ionosphere will be broader with larger densities than the night side ionosphere. The night side ionosphere will begin at higher altitudes above Titan's surface. The model results shown in Fig. 3 give ion densities in good agreement with Cassini and Voyager observations (cf. Keller et al., 1998; Cravens et al., 2004). They are however about a factor of 2 larger at lower altitudes than those reported by Ågren et al. (2009). Indeed several ionospheric models produce densities that are too large. This problem was pointed out by Ågren et al. (2007). Understanding the complexity of Titan's ionosphere is an ongoing challenge. Recent results from Galand et al. (2010) suggest that the effective recombination rates increase to values higher than previously expected with decreasing altitudes. Galand et al. (2010) point out that more laboratory measurements of electron dissociative recombination coefficients are needed to improve our ability to model the deep 
Table 1. The generic ion species used in the model, the actual ion species they represent, their assigned mass and the mass range of the actual ions represented.

\begin{tabular}{|c|c|c|c|}
\hline Name & Components & Mass (amu) & Mass range \\
\hline $\mathrm{L}^{+}$ & $\mathrm{H}^{+}, \mathrm{H}_{2}{ }^{+}, \mathrm{H}_{3}{ }^{+}$ & 2 & $1-3$ \\
$\mathrm{M}^{+}$ & $\mathrm{CH}_{5}{ }^{+}, \mathrm{N}^{+}, \mathrm{CH}_{4}{ }^{+}, \mathrm{CH}_{3}{ }^{+}, \mathrm{CH}_{2}{ }^{+}, \mathrm{CH}^{+}, \mathrm{C}^{+}$ & 14 & $12-17$ \\
$\mathrm{H}^{+}$ & $\mathrm{C}_{2} \mathrm{H}_{5}{ }^{+}$ & 29 & 29 \\
$\mathrm{H}^{+}$ & $\mathrm{CHNH}^{+}$ & 28 & 28 \\
$\mathrm{MHC}^{+}$ & $\mathrm{C}_{3} \mathrm{H}^{+}, \mathrm{C}_{3} \mathrm{H}_{2}{ }^{+}, \mathrm{C}_{3} \mathrm{H}_{3}{ }^{+}, \mathrm{C}_{3} \mathrm{H}_{4}^{+}, \mathrm{C}_{3} \mathrm{H}_{5}{ }^{+}, \mathrm{C}_{4} \mathrm{H}_{3}{ }^{+}, \mathrm{C}_{4} \mathrm{H}_{5}{ }^{+}$ & 44 & $37-53$ \\
$\mathrm{HHC}^{+}$ & $\mathrm{C}_{5} \mathrm{H}_{3}{ }^{+}, \mathrm{C}_{5} \mathrm{H}_{5}{ }^{+}, \mathrm{C}_{5} \mathrm{H}_{7}^{+}, \mathrm{C}_{5} \mathrm{H}_{9}{ }^{+}, \mathrm{C}_{6} \mathrm{H}_{5}{ }^{+}, \mathrm{C}_{6} \mathrm{H}_{7}{ }^{+}, \mathrm{C}_{7} \mathrm{H}_{5}{ }^{+}$ & 70 & $68-89$ \\
$\mathrm{HNI}^{+}$ & $\mathrm{C}_{3} \mathrm{H}_{2} \mathrm{~N}^{+}, \mathrm{C}_{5} \mathrm{H}_{5} \mathrm{~N}^{+}, \mathrm{C}_{3} \mathrm{HN}^{+}$ & 74 & $51-79$ \\
\hline
\end{tabular}

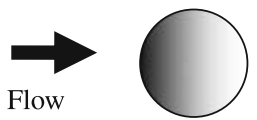

Case 1

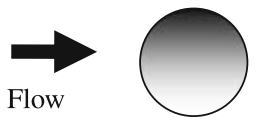

Case 2

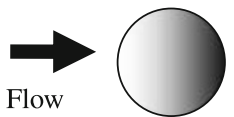

Case 3

Fig. 4. This is a schematic showing the orientation of the sunlit hemisphere with respect to the incident flow. The incident magnetic field points into the diagram.

ionosphere. Incorporating larger recombination rates in the model will decrease the ionospheric densities and reduce the effective obstacle size created by the ionosphere.

Titan's lower ionosphere has been found to be dominated (between 10-60\%) by heavy (greater than $100 \mathrm{amu}$ ) organic compounds (cf. Crary et al., 2009; Wahlund et al., 2009). These compounds are currently represented in our model by the $\mathrm{HHC}^{+}$and $\mathrm{HNI}^{+}$species. These species contain a representation of all the heavy and very heavy hydrocarbon and nitrile ion species and related chemistry. They provide a fair representation of this part of the ionosphere for the purposes of this study. The ionospheric model used in the simulations is an initial step that will be refined as understanding of Titan's ionosphere improves. Further details about the model can be found in Appendix A.

The ion-neutral chemical model is implemented into the hybrid code using a spherical grid similar to the grid used for the neutral collisions described above. The density of each ion species is volumetrically weighted to the cell corners. The ion-neutral chemistry is then integrated explicitly. At the end of the integration time, 1 particle is added for each species in every cell of the chemistry grid. This particle is weighted such that it represents the total density created in that cell during the integration time. The weights of the old particles of a given species located in the cell are reduced to account for losses due to recombination and chemical reactions. At the start of each simulation the ion-neutral chemistry is evolved for $20000 \mathrm{~s}$ to ensure that chemical equilibrium is reached to an altitude of $2000 \mathrm{~km}$. This is well above the altitude where the advection time scales are equal to the net ion production time scales. The ionosphere is over built at start up, it then erodes until a balance is reached between the rate the ions are produced and the rate they are being advected away. This approach avoids the po-
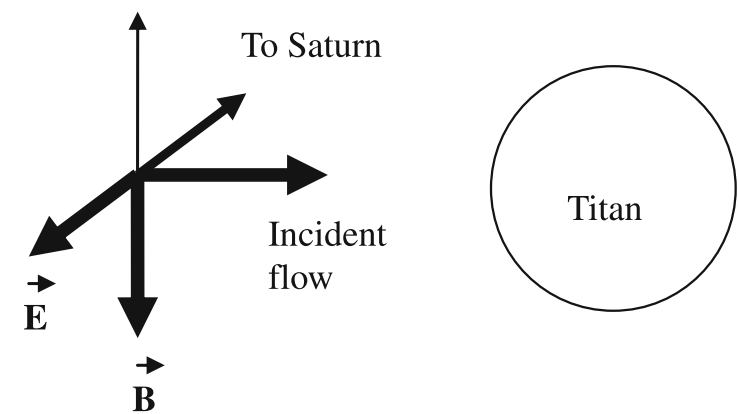

Fig. 5. This is a schematic representation of the directions of the incident electric and magnetic fields with respect to the flow direction and Saturn. The incident flow is in the direction of the $\mathbf{E} \times \mathbf{B}$ drift.

tential problem of the ions being lost to Saturn's magnetosphere before the ionosphere has a chance to build. Hence a fully developed ionosphere is present when the run begins. While the simulations are running the chemistry package is able to accurately respond to changes in the ion densities.

\section{The Simulations}

Three simulations with the day side hemisphere located in different orientations with respect to Saturn's magnetospheric flow were run. Figure 1 shows a schematic representation of where Titan is along its orbit and the orientation of the sunlit hemisphere with respect to the plasma flow for each case. The sun is to the left, Saturn is at the center. Case 1 occurs when Titan is at 6:00 Saturn local time (SLT), case 2 occurs when Titan is at 12:00 and case 3 occurs when Titan is located at 18:00 SLT. A close up view showing the orientation of the sunlit hemisphere with respect to the flow direction and Saturn is also shown in Fig. 4.

Figure 5 is a schematic showing the three-dimensional 
Polar View

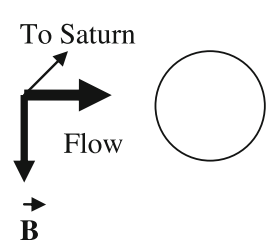

Orbital View

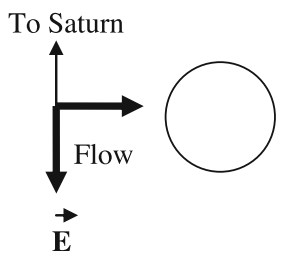

Projected View

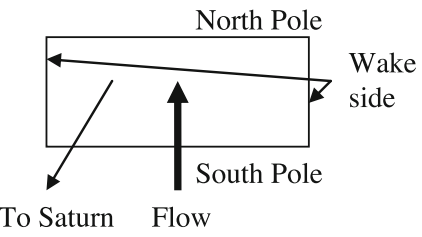

A

B

C

Fig. 6. Sketches of the three views used to present the simulation results. (A) The polar view is the plane that contains the flow direction and the incident magnetic field. Saturn is located into the page. (B) The orbital view is Titan's orbital plane. The plane contains the convection electric field and the flow. Saturn is located to the top in this view. (C) The projected view is the projection of an unwrapped sphere into a two-dimensional plane. The incident flow is into the center of the plane $\left(0^{\circ}\right.$ latitude, $0^{\circ}$ longitude). Saturn is in the direction normal to the plane at $-90^{\circ}$ latitude and $0^{\circ}$ longitude. The bottom edge represents the south pole, the top edge the north pole, while the right and left edges meet on the back side.

Table 2. The net ion loss rates for each species.

\begin{tabular}{|c|c|c|c|c|}
\hline Species & AMU & Case 1 & Case 2 & Case 3 \\
\hline $\mathrm{L}^{+}$ & 2 & $3.0 \times 10^{21}$ & $3.0 \times 10^{21}$ & $2.3 \times 10^{21}$ \\
$\mathrm{M}^{+}$ & 14 & $1.7 \times 10^{24}$ & $1.7 \times 10^{24}$ & $1.5 \times 10^{24}$ \\
$\mathrm{H}^{+}$ & 29 & $3.0 \times 10^{24}$ & $3.2 \times 10^{24}$ & $1.9 \times 10^{24}$ \\
$\mathrm{H}^{+}$ & 28 & $7.0 \times 10^{23}$ & $6.9 \times 10^{23}$ & $6.4 \times 10^{23}$ \\
$\mathrm{MHC}^{+}$ & 44 & $2.8 \times 10^{23}$ & $2.7 \times 10^{23}$ & $2.7 \times 10^{23}$ \\
$\mathrm{HHC}^{+}$ & 70 & $5.7 \times 10^{22}$ & $6.1 \times 10^{22}$ & $6.6 \times 10^{22}$ \\
$\mathrm{HNI}^{+}$ & 74 & $2.0 \times 10^{19}$ & $9.1 \times 10^{19}$ & $1.2 \times 10^{19}$ \\
\hline
\end{tabular}

orientation of the magnetic field, the convection electric field, the plasma flow direction and the direction to Saturn. The incident magnetic field points southward (down in the figure), the convection electric field points away from Saturn; the plasma flow is from the left. The plasma flow is in the direction of the $\mathbf{E} \times \mathbf{B}$ drift.

There are three points of view that will be used in this paper to present the simulation results. Each of the points of view is illustrated in Fig. 6. The polar view (6(A)) is the plane that contains the plasma flow and the incident magnetic field. The direction of Saturn is into the page. The orbital view (6(B)) is a cut in Titan's orbital plane. The orbital plane contains the convection electric field and the plasma flow. Saturn is located at the top of this view. The projected view $(6(C))$ is the projection of an unwrapped sphere into a two-dimensional plane. The incident flow is into the center of the plane $\left(0^{\circ}\right.$ latitude, $0^{\circ}$ longitude). Saturn is in the direction normal to the plane at $-90^{\circ}$ latitude, $0^{\circ}$ longitude. The bottom edge represents the south pole, the top edge the north pole, while the right and left edges meet on the back side.

The plasma and field conditions are the same for each case. The magnetic field is taken to be $5 \mathrm{nT}$ pointing southward perpendicular to Titan's orbital plane. We take the incident plasma to consist of only $\mathrm{O}^{+}$with a density of $0.2 \mathrm{~cm}^{-3}$ moving with a speed of $120 \mathrm{~km} / \mathrm{s}$ with respect to Titan. The incident electrons have a density of $0.2 \mathrm{~cm}^{-3}$ and a temperature of $200 \mathrm{eV}$. We do not address the issue of the upstream ion distribution function but adopt the simplest approach and load the ions with a beam distribution.

\section{Results}

\subsection{Ion loss rates}

There are two straightforward questions that the simulation results can be used to answer. The first is how much of each ion species is Titan losing to Saturn's magnetosphere? The second is does the orientation of the sun with respect to the plasma flow direction effect those loss rates? To address these question we constructed a $5 R_{\mathrm{T}}$ sampling box centered on Titan. Ions that crossed the sampling faces where collected over the time of each run and recorded. The loss rates were then calculated. Each simulation was run until the loss rates became stable. All of the cases took longer than $2700 \mathrm{~s}$ of simulation time (not run time) for the loss rates to stabilize. Rates collected before that time overestimated the loss of $\mathrm{L}^{+}$and $\mathrm{M}^{+}$. All of the species except the $\mathrm{HNI}^{+}(74 \mathrm{amu})$ first hit the sampling box within 500 seconds, with $\mathrm{L}^{+}, \mathrm{M}^{+}, \mathrm{H}^{+}$and $\mathrm{H}_{2}^{+}$all hitting in less than $100 \mathrm{~s}$. The $\mathrm{MHC}^{+}$first hit the box around $250 \mathrm{~s}$ while the $\mathrm{HHC}^{+}$took almost $500 \mathrm{~s}$. The $\mathrm{HNI}^{+}$took more than twice as long, over $1300 \mathrm{~s}$ to reach the box. The lower the altitude of the peak density for a given species, the longer it takes that species to escape and be sampled at $2.5 R_{\mathrm{T}}$.

The final estimates of the loss rates of each species for each case are shown in Table 2. The total ion loss rates for each case are: $5.7 \times 10^{24} \mathrm{~s}^{-1}, 5.9 \times 10^{24} \mathrm{~s}^{-1}$ and 4.3 $\times 10^{24} \mathrm{~s}^{-1}$ for cases 1,2 and 3 respectively. The rates (both the total and by species) are fairly consistent with most values being well within a factor of two for each case. The exception being the loss rates for the $\mathrm{HNI}^{+}$where the spread is a bit larger. A graphical representation of the loss rates is given in Fig. 7 .

Figure 7 shows the loss rate as a function of the species 
Loss Rate and Altitude of the Ionspheric Density Peak vs. Species Mass

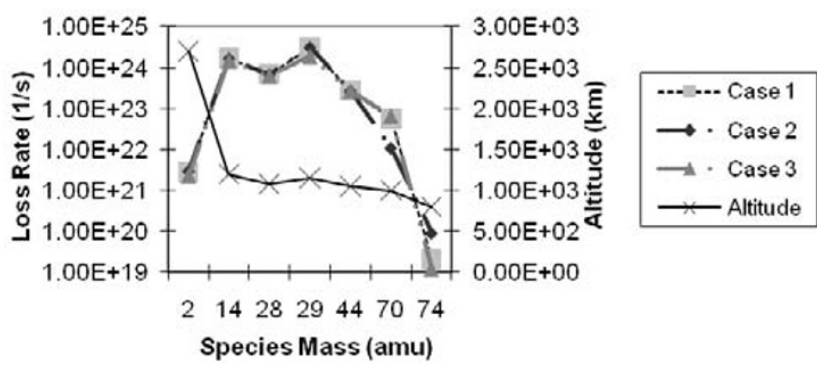

Fig. 7. This plot shows the loss rate as a function of the species mass for each case. The altitude of the dayside ionospheric density peak for each species is also plotted. The loss rates for a given species are fairly consistent. For most of the masses case 2 is the orientation that leads to the largest ion escape, though only slightly. There is also an altitude trend for most of the masses. The more massive the species, the lower the altitude of its ionospheric density peak and the lower its loss rate to Saturn's magnetosphere is.

mass for each case. The altitude of the dayside ionospheric density peak for each species is also plotted. The loss rates for a given species are fairly consistent. For most of the masses case 2 is the orientation that leads to the largest ion escape, though only slightly. There is also an altitude trend for most of the masses. The more massive the species, the lower the altitude of its ionospheric density peak and the lower its loss rate to Saturn's magnetosphere is. The exception to that trend is the $\mathrm{L}^{+}$species. It's interesting to note that the loss rates for the $\mathrm{H}^{+}{ }^{+}$and $\mathrm{H}_{2}{ }^{+}$are different even though their masses are about the same (29 versus 28 $\mathrm{amu}$ ). They should respond to the electric and magnetic fields in a very similar fashion. So the expectation that their loss rates should be similar would not be unreasonable. They are not shown here but the particle trajectories for each species outside of the ionosphere are very similar. The difference in their loss rates is a result of their creation. The ion density profiles in Fig. 3 show that $\mathrm{H}^{+}$is more abundant than $\mathrm{H}_{2}^{+}$in the upper ionosphere. Therefore there is more of it for Saturn's magnetosphere fields to pick up.

Figure 8 shows the log of the net radial ionospheric ion flux through a sphere at $2500 \mathrm{~km}$ altitude for each case. Note in this figure a negative value means the flux is towards Titan. In all of the cases the inward and outward radial fluxes are similar in value and angular coverage. Ion escape is out the anti-Saturn hemisphere and the poles in each case regardless of the orientation of the dayside ionosphere.

\subsection{Ion densities}

The projected view of the final $\mathrm{H}^{+}$density at $1200 \mathrm{~km}$ altitude is shown in Fig. 9. The orientation of the day and night side ionospheres is evident. There is a solar zenith angle variation in the dayside density, with no angular dependence in the nightside density. These variations are a result of the solar zenith angle dependence of the photoionization rates (see Appendix A). The results are in agreement with the photochemical results shown in Fig. 3. Thus the ionosphere is in photochemical equilibrium at this altitude.

The initial $\mathrm{H}^{+}$densities for each case at $1300 \mathrm{~km}$ altitude are shown in Fig. 10. The densities range from
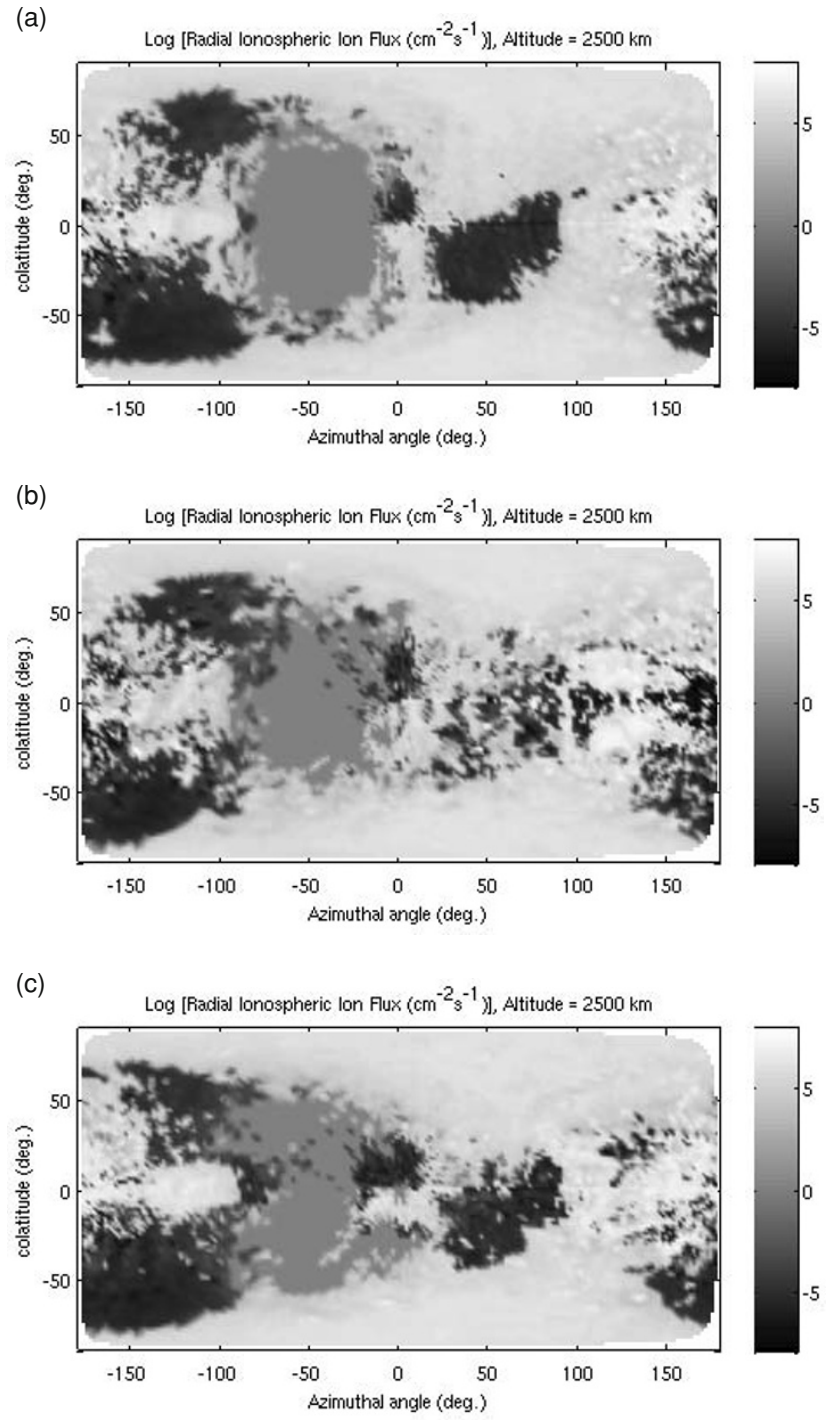

Fig. 8. This figure shows the Log of the net radial ionospheric ion flux through a sphere at $2500 \mathrm{~km}$ altitude for each case, case 1 (A), case 2 (B) and case 3 (C). Negative values indicate the radial ion flux is directed towards Titan. Qualitatively each the results for each case are similar to each other. The ions are escaping from the poles and the anti-Saturn facing hemisphere. Ions are moving toward titan in the Saturn facing hemisphere. There is little ionospheric ion flux through the downstream quadrant of the Saturn facing hemisphere.

$800 \mathrm{~cm}^{-3}$ on the dayside down to $300 \mathrm{~cm}^{-3}$ in the nightside hemisphere. In contrast the final $\mathrm{H}^{+}$densities are shown in Fig. 11 show significant ion loss. The peak dayside ionospheric density is now down to about $225 \mathrm{~cm}^{-3}$ with the nightside densities dropping to around $100 \mathrm{~cm}^{-3}$. The ionosphere is no longer in photochemical equilibrium at this altitude. The initial density has been eroded away by Saturn's magnetosphere. There is a $10-15 \mathrm{~cm}^{-3}$ (or $4 \%-$ $6 \%$ ) variation in the dayside ionospheric density between the cases.

Contours of the $\log \left(n_{e}\right)$ in the polar view for each run are shown in Fig. 12. There is an increase in the density upstream of Titan forming a bow wave. The position, shape and density jump of the bow wave does not depend on the illumination angle. The maximum density in each plot occurs in the dayside ionosphere at an altitude of $1100 \mathrm{~km}$, 
(a)

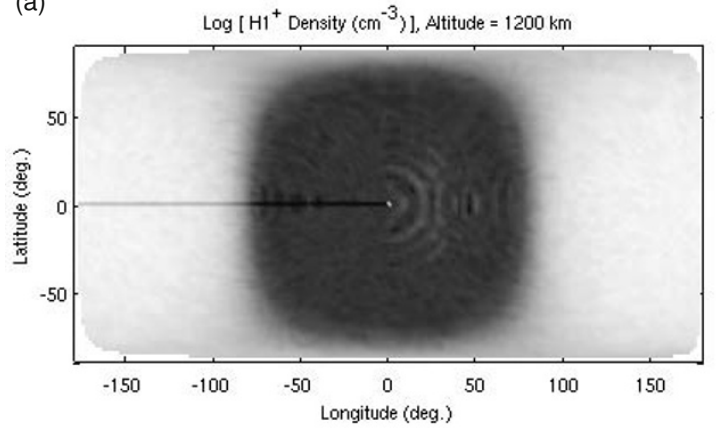

(b)

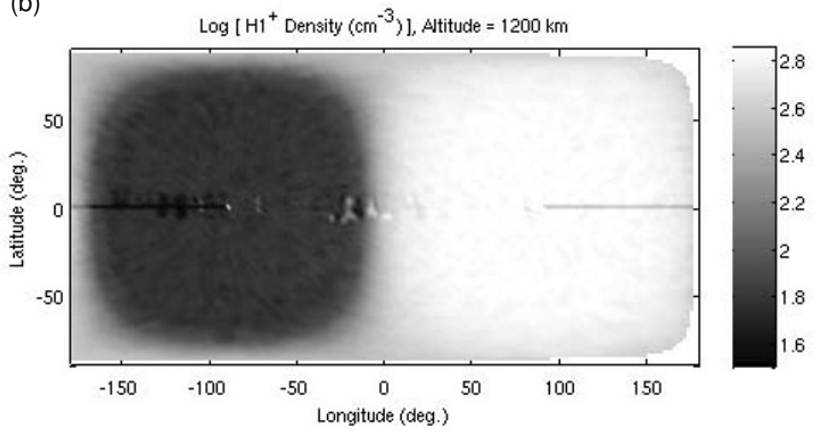

(c)

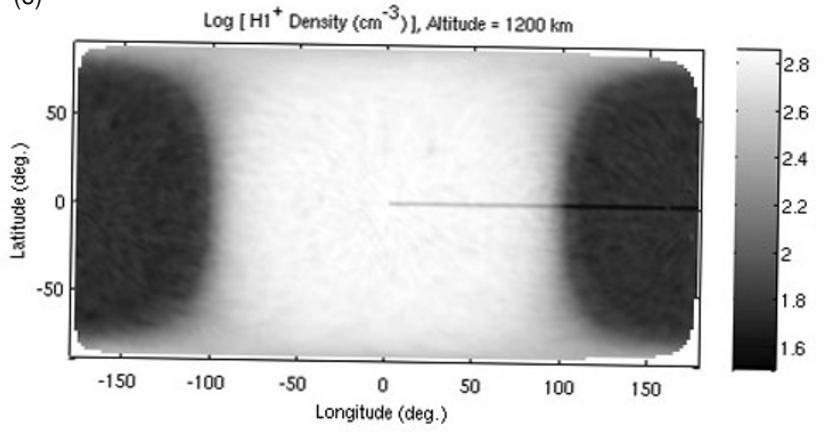

Fig. 9. The $\log$ of the final $\mathrm{H}^{+}$densities for case 1 (A), case 2 (B) and case $3(\mathrm{C})$ at $1200 \mathrm{~km}$ altitude are shown. The densities are represented by $10 \mathrm{~s}$ of thousands of particles in each cell of the chemistry grid. The results show good agreement with the 1-dimensional photochemical results shown in Fig. 2 indicating that the ionosphere is in photochemical equilibrium at this altitude.

with a value around $3200 \mathrm{~cm}^{-3}$. This is in reasonable agreement (within a factor of 2) with the observed electron densities and 1-dimensional results shown in Fig. 3. This density is present throughout the duration of each simulation, indicating that the ionosphere is being maintained by the ion-neutral chemistry and ion-neutral collisions. The density tail is confined to with $\pm 1 R_{\mathrm{T}}$ in each case. Each case shows the density being focused downstream of Titan before diverging. The downstream location of the focal point is about $2-3 R_{\mathrm{T}}$ further downstream in case 1 than it is in the other two cases.

The contours of $\log \left(n_{e}\right)$ in the orbital view for each case are shown in Fig. 13. The results show a density tail that is deflected from the magnetospheric flow direction. The bow wave in this plane is asymmetric about the flow axis and shows no dependence on the illumination angle. Plasma streams and blobs can be seen coming off of the ionosphere. There is a sharp density transition on the Saturn facing side (a)
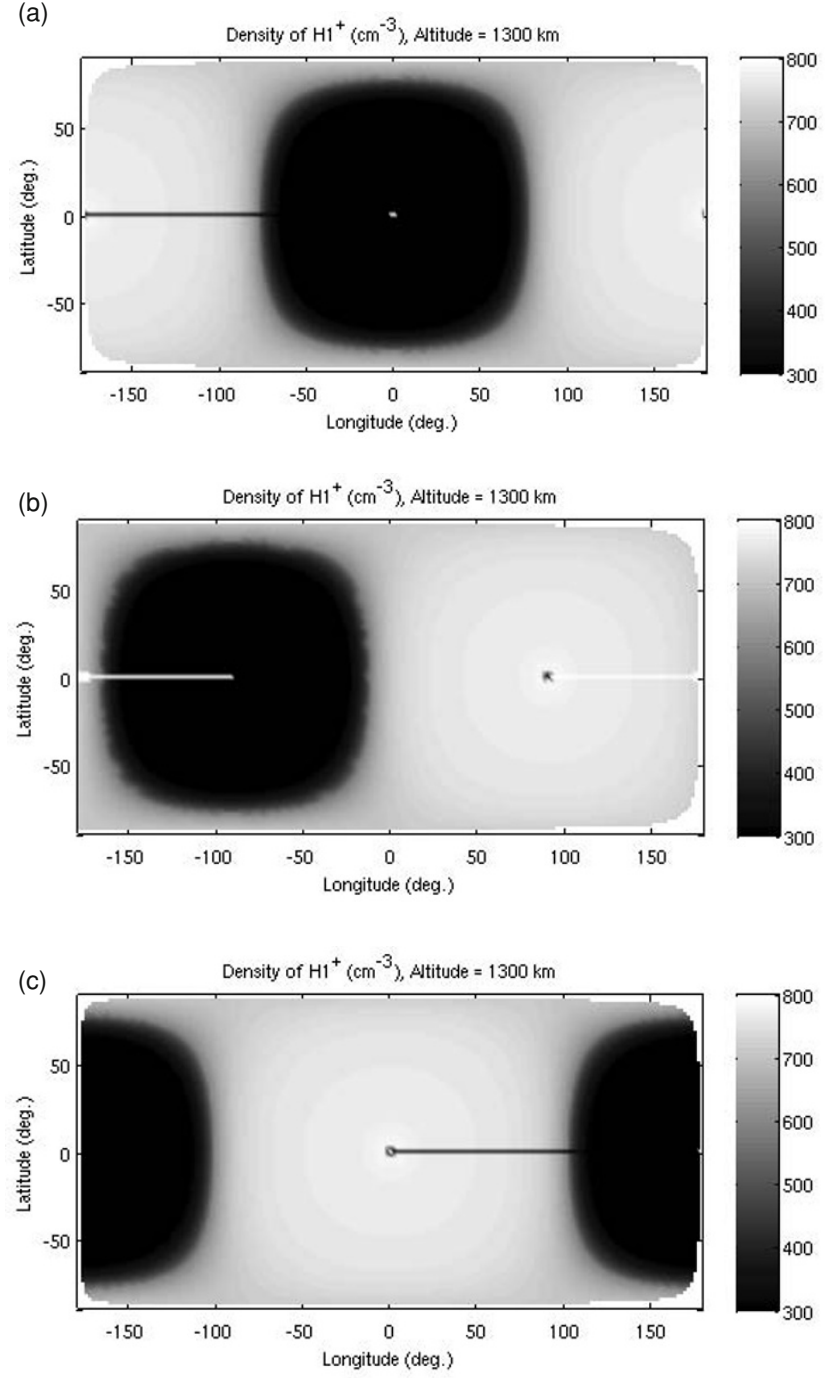

Fig. 10. The initial $\mathrm{H}^{+}$densities at an altitude of $1300 \mathrm{~km}$ for each simulation are shown, case 1 (A), case 2 (B) and case $3(\mathrm{C})$.

of the interaction. The density transition is more diffuse on the anti-Saturn facing side. Blobs of plasma can be seen in this side. These are transient features that can affect other aspects of the simulation such as the fields. The illumination angle does have an effect on where the plasma blobs detach from Titan's ionosphere and their trajectories afterwards. When the dayside ionosphere is on the anti-Saturn facing side of Titan the plasma blobs originate from the upstream side of Titan. They move out the flank region on cycloidal trajectories with large gyroradii. Their trajectories resemble the test-particle trajectories of reported by Luhmann (1996) and Ledvina et al. (2000). In contrast when the dayside ionosphere is along the ram or wake hemispheres as in cases 1 and 3, the ions are moved more tail ward. These are transient features; they don't have significant effect on the global features of the interaction.

The log of the magnetospheric $\mathrm{O}^{+}$density in the orbital plane for each run is shown in Fig. 14. There is a bow wave resulting in a density buildup upstream of Titan present in each case. The bow wave is not symmetric about the flow axis. The density build up across the wave and its standoff distance from Titan increases as the illumination angle 
(a)

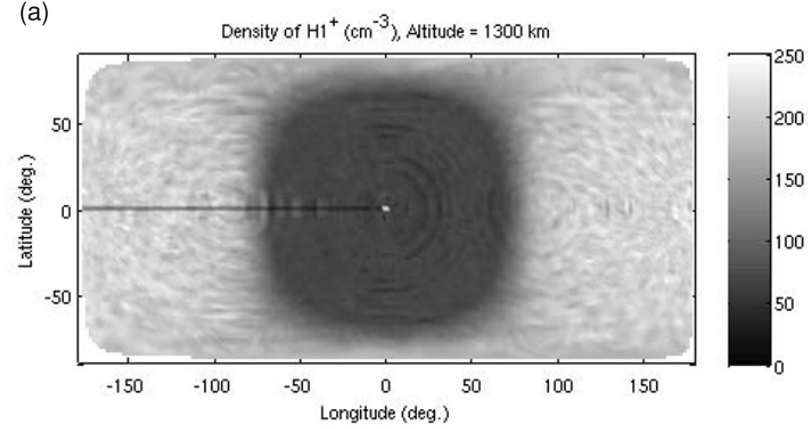

(b)

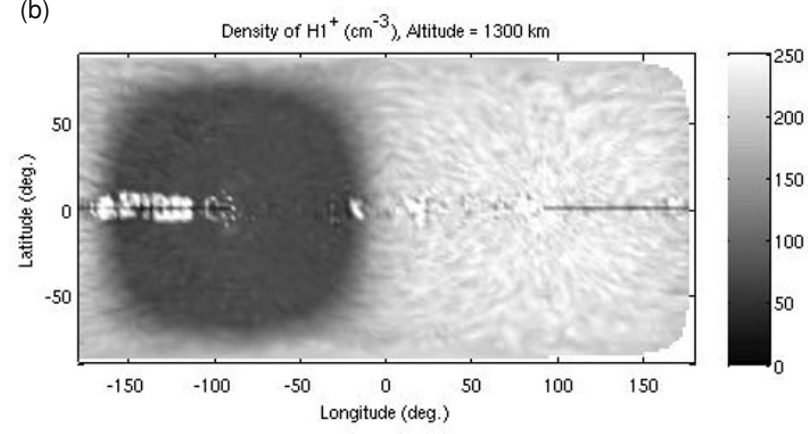

(c)

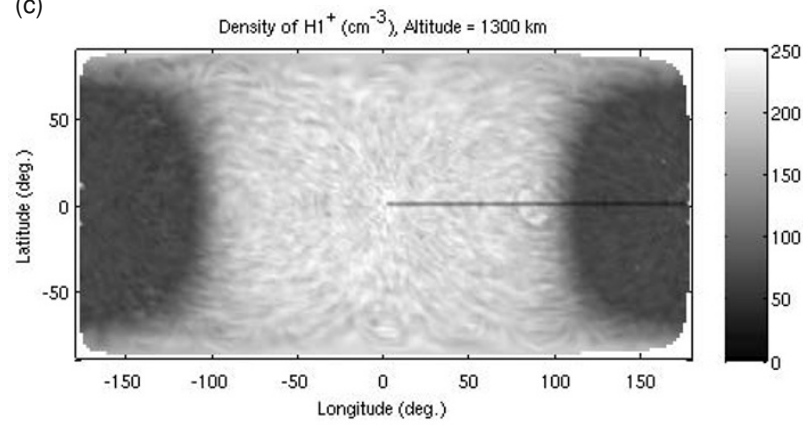

Fig. 11. The final $\mathrm{H}^{+}{ }^{+}$densities for case $1(\mathrm{~A})$, case 2 (B) and case 3 (C) at $1300 \mathrm{~km}$ altitude are shown. The densities are lower than the initial densities shown in Fig. 10 indicating that the ionosphere is no longer in photochemical equilibrium at this altitude. Dynamical processes are not important.

with respect to the sub-flow point goes to zero. As the subsolar point becomes aligned with the subflow point more ionospheric plasma is built up on the upstream hemisphere of Titan. This gives Saturn's magnetosphere more plasma to interact with accounting for the differences in the bow waves between the cases. Each case shows a density drop out of $\mathrm{O}^{+}$on the wake side that corresponds to the density streams in Fig. 13. There is a density build up along the Saturn-facing side of the wake region. The turbulent densities in the anti-Saturn side of the interaction correspond to the plasma blobs present in Fig. 13.

There is no significant $\mathrm{L}^{+}$build up in Titan's ionosphere for any of the simulation results (see Fig. 15). There are streams and ion blobs of $\mathrm{L}^{+}$in the wake regions. The densities of these streamers are small compared to the other species. However, there are places where they are still factors of 2-3 times larger than the incident $0.1 \mathrm{~cm}^{-3} \mathrm{H}^{+}$reported by Voyager and an order of magnitude larger than some of the values reported by Cassini. It's important to note that this was not the case at the start of each simu-
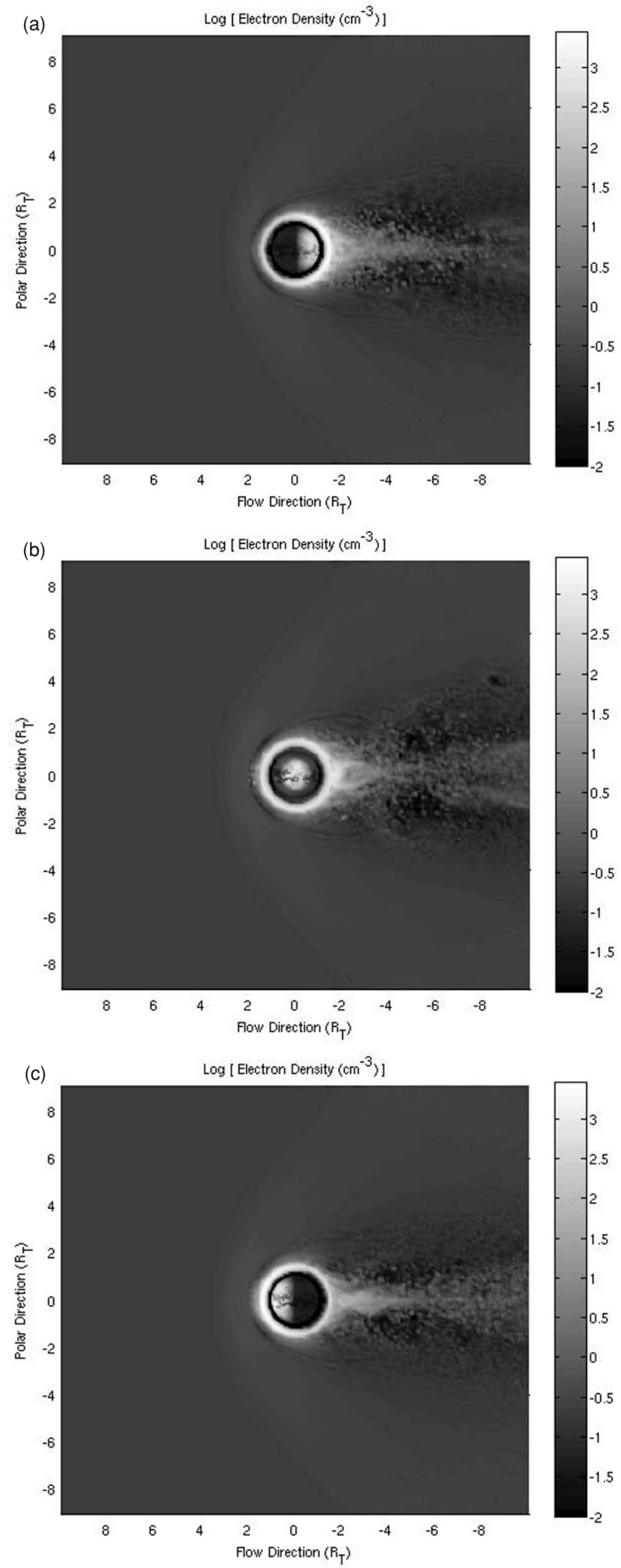

Fig. 12. The polar view showing contours of the Log of the electron densities for case 1 (A), case 2 (B) and case 3 (C) are shown. The density in each case forms a current sheet in the wake.

lation. The initial ionosphere built via the chemistry created a halo of $\mathrm{L}^{+}$around Titan with a density ranging $\sim 8$ $20 \mathrm{~cm}^{-3}$. This halo was eroded away by Saturn's magnetosphere faster than the chemistry could replenish it. 

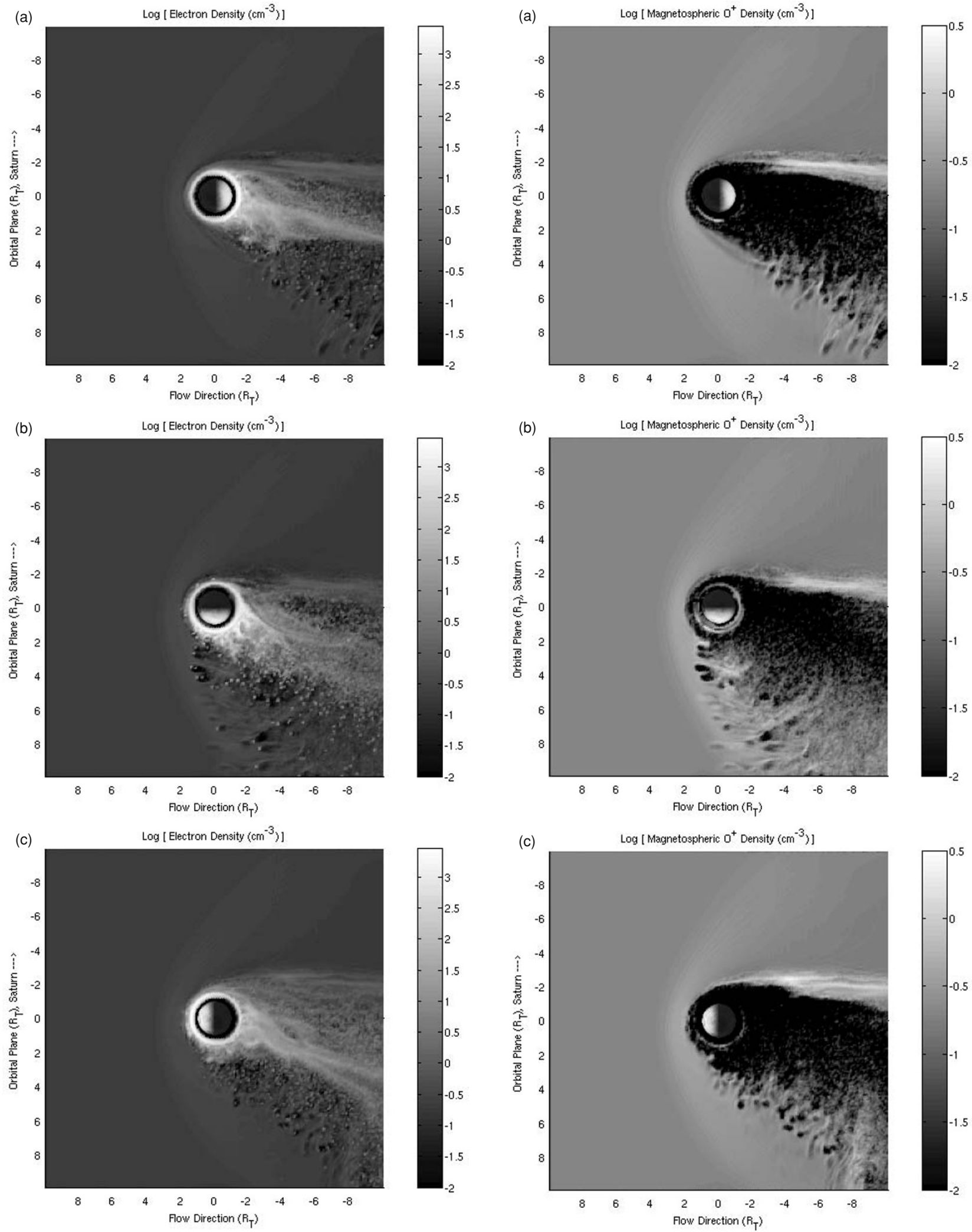

Fig. 13. Contours of $\log \left(n_{e}\right)$ in the orbital view for case 1 (A), case 2 (B) and case $3(\mathrm{C})$.

Contours of the $\log$ of the $\mathrm{M}^{+}$density show a lot of structure in the near Titan region (see Fig. 16). The results contain plasma blobs detaching from the ionosphere and moving downstream. The results also contain streams of plasma coming off the ionosphere. The location of the

Fig. 14. Contours for the $\log \left(n_{\mathrm{O}^{+}}\right)$in the orbital view for case 1 (A), case 2 (B) and case $3(\mathrm{C})$. There is a bow wave resulting in a density buildup upstream of Titan present in each case. Each case shows a density drop out on the wakeside that corresponds to the density streams shown in Fig. 13. There is a density build up along the Saturn-facing side of the wake region for each case. The turbulent densities in the anti-Saturn side of the interaction correspond to the plasma blobs present in Fig. 13. 

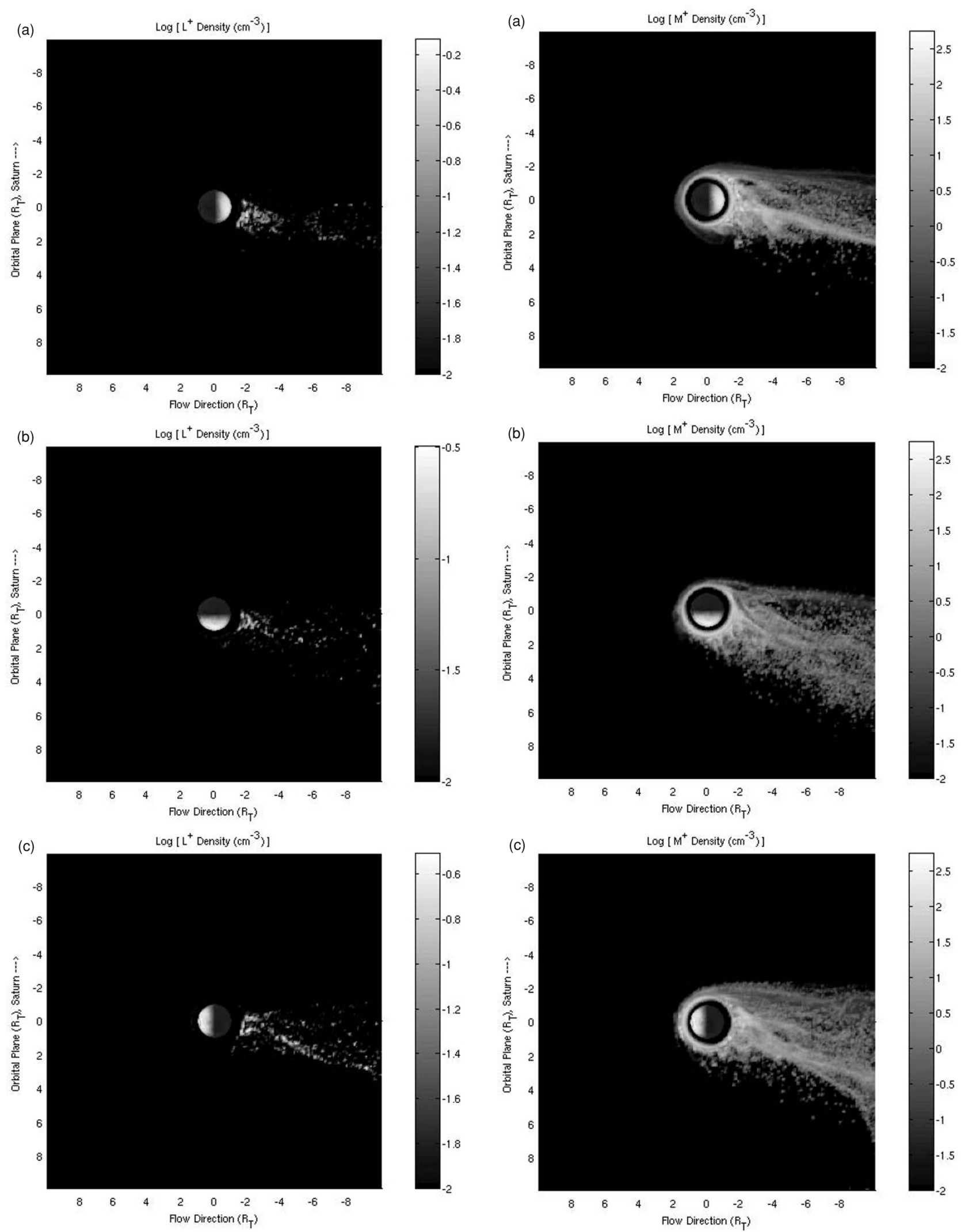
Fig. 15. Contours of $\log \left(n_{\mathrm{L}^{+}}\right)$in the orbital plane for each case are shown,
case 1 (A), case 2 (B) and case 3 (C). There is no significant $\mathrm{L}^{+}$density present in Titan's ionosphere in any of the results. However streams of $\mathrm{L}^{+}$are found in the wake region.

Fig. 16. Contours of $\log \left(n_{\mathrm{M}^{+}}\right)$in the orbital plane for case 1 (A), case 2 (B) and case 3 (C) are shown. 

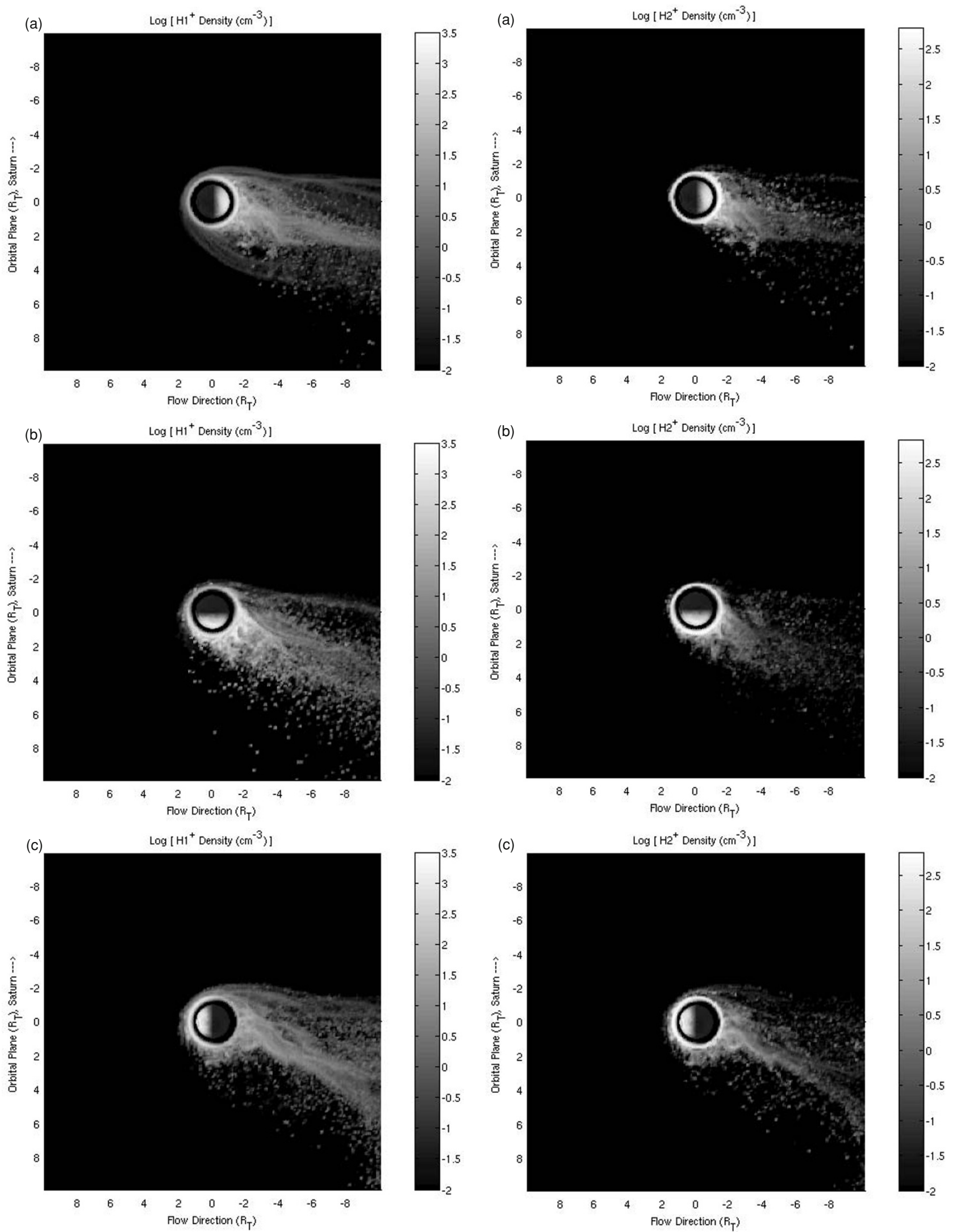

Fig. 17. Contours of the $\log \left(n_{\mathrm{H} 1^{+}}\right)$in the orbital plane for run 1 (A), run 2 (B) and run $3(\mathrm{C})$ are shown.

Fig. 18. Contours for the $\log$ of the $28 \mathrm{amu} \mathrm{H} 2^{+}$for case 1 (A), case 2 (B) and case 3 (C) are shown. 

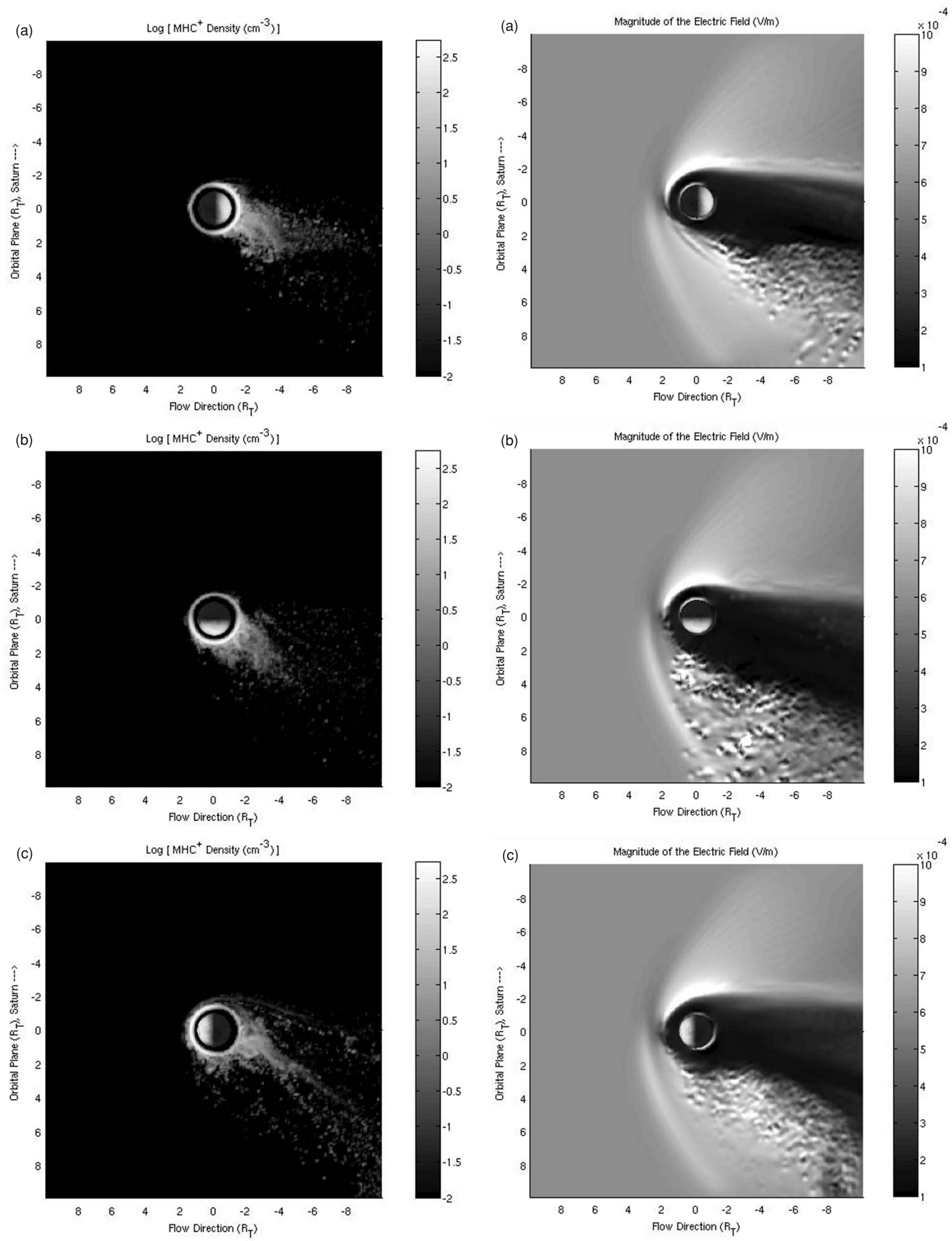

Fig. 19. Contours of the log of the $\mathrm{MHC}^{+}$species for case 1 (left), case 2 (middle) and case 3 (right).

Fig. 20. The magnitude of the electric field in the orbital plane for each run, case 1 (left), case 2 (middle) and case 3 (right). 


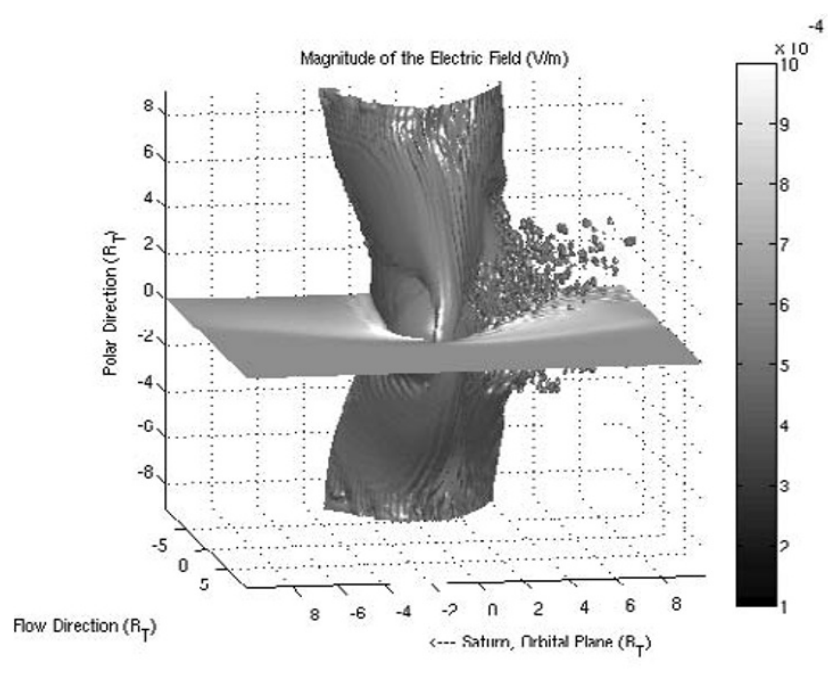

Fig. 21. The $5 \times 10^{-4} \mathrm{~V} / \mathrm{m}$ isosurface of the electric field for case 1 is shown. The flow is into the page and Saturn is to the left. There is a twist in the field at the sub-flow point.

density structures in the wake region are in agreement with the electron density structures in the corresponding regions of Fig. 13. More than half of the plasma in the density streamers in Fig. 13 is made up of $\mathrm{M}^{+}$. The width of the plasma tail is slightly wider than the width of Titan's ionosphere.

It is interesting to compare and contrast the density contours for the $\mathrm{H}^{+}$and $\mathrm{H}_{2}^{+}$species shown in Figs. 17 and 18. There is only 1 amu difference in the mass of each species. It might be expected then that both species should behave similarly with comparable density structures and values. That is not the case however. It is clear from the figures that there is much more $\mathrm{H}^{+}{ }^{+}(29 \mathrm{amu})$ being lost from Titan than the $\mathrm{H}_{2}{ }^{+}(28 \mathrm{amu})$. This is in agreement with the results shown in Table 2 and Fig. 7. There are similarities in the locations of the density streamers for each species and the width of the tails. These are features that are associated with the motion of the ions. These ion species have similar masses so the locations of these features are similar for both species. However their densities are not. As mentioned in the previous section this is due to the fact that more $\mathrm{H}^{+}$ than $\mathrm{H} 2^{+}$is created in the upper ionosphere by the chemistry.

The density results for the $\mathrm{MHC}^{+}$species (44 amu) are shown in Fig. 19. The density streamers dissipate much closer to Titan than the streamers composed of the lighter ion species. As they dissipate they break up into blobs of plasma and spread out.

The results for the heaver species $\left(\mathrm{HHC}^{+}, 70 \mathrm{amu}\right.$ and $\left.\mathrm{HNI}^{+}, 74 \mathrm{amu}\right)$ are not shown here. Very little of these species are lost from the ionosphere. What does manage to escape leaves as very thin plasma streams that quickly break up into blobs of plasma. There is little difference between the density structures of these two species or the between structures in each of the cases.

\subsection{The electric and magnetic fields}

The magnitude of the electric field in the orbital plane for each run is shown in Fig. 20. To first order the elec-
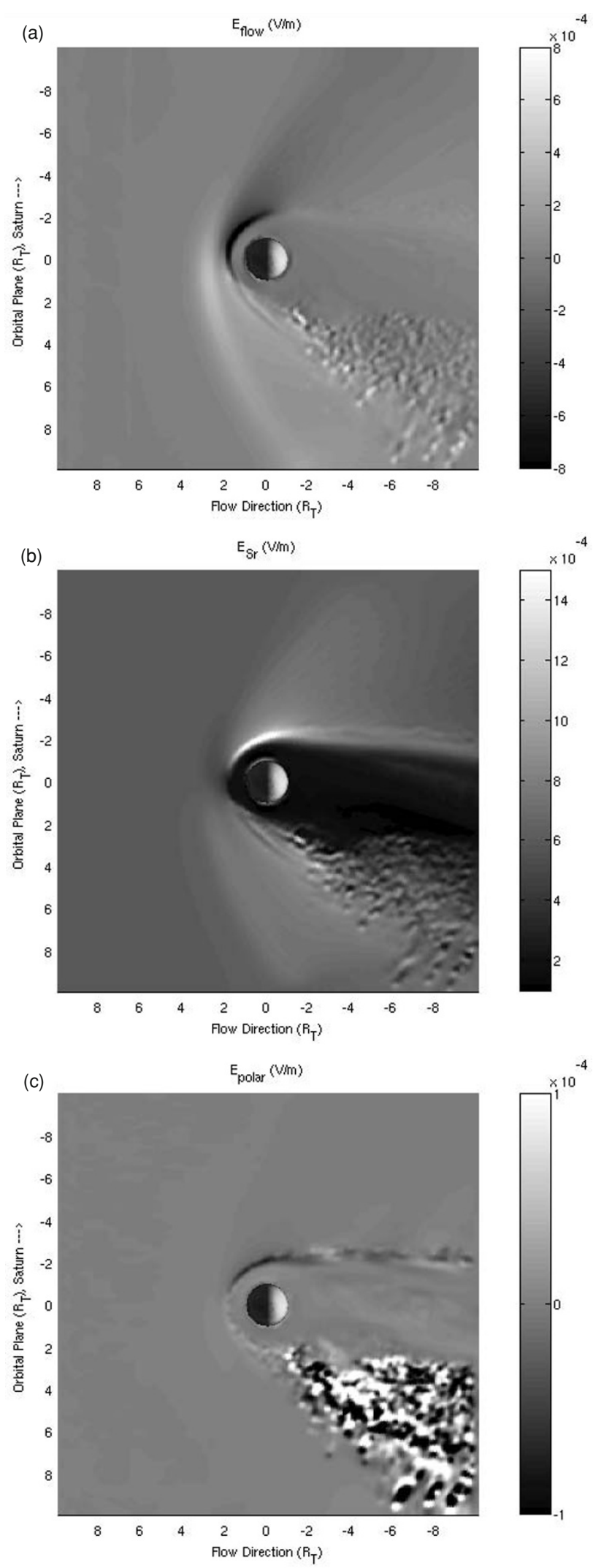

Fig. 22. The magnitude of the electric field components for case 1 in the orbital plane (A) along the flow direction, (B) along the convection electric field direction and (C) perpendicular to the orbital plane are shown. 

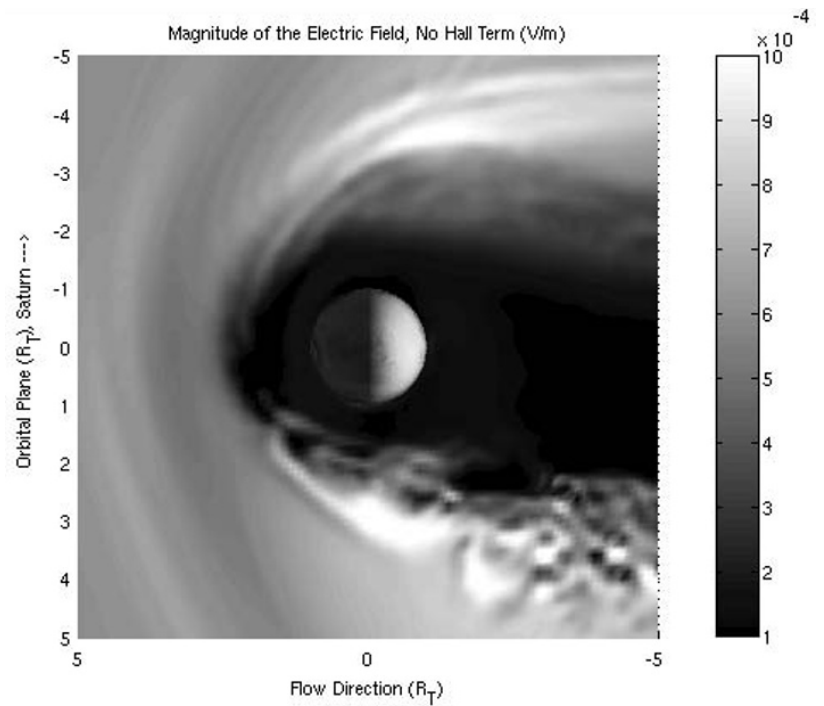

Fig. 23. Contours of the magnitude of the electric field around Titan for case 1 with the Hall term removed are shown. The contours are more symmetric about the flow axis without the Hall term than they are in Fig. 20(a).

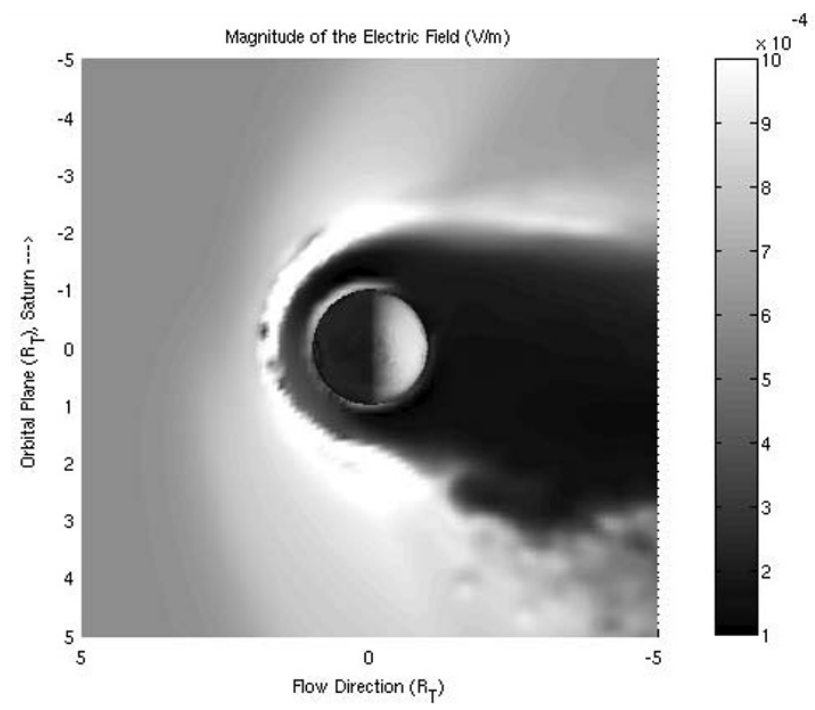

Fig. 24. The contours of the magnitude of the electric field for case 1 with the $\nabla P_{e}$ term removed from the simulation.

tric field results are the same. The corotational electric field has a value of $6 \times 10^{-4} \mathrm{~V} / \mathrm{m}$. There is an increase in the field strength across the bow wave $\left(\sim 8 \times 10^{-4} \mathrm{~V} / \mathrm{m}\right)$. A large increase in the field strength occurs in the Saturn facing hemisphere near Titan. The electric field build up, runs along the Saturn facing side of the wake region. The field strength drops in the wake region behind Titan. Each run shows a discontinuity in the field strength in the sub-flow point upstream of Titan. The fields are not symmetric about the plasma flow axis. There is significant wave activity in the anti-Saturn side of the interaction. This wave activity is associated with the pickup ions and plasma blobs. The location of the wave activity with respect to Titan is different in each case, depending on the location of the dayside ionosphere.

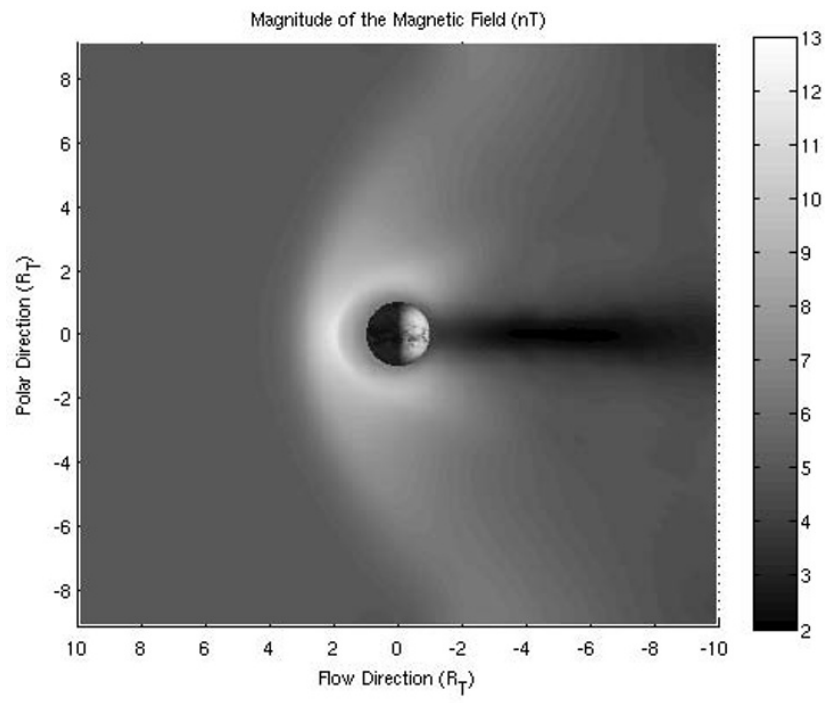

Fig. 25. Polar view of the magnitude of the magnetic field around Titan for case 2 is shown. There is a buildup in the field strength to about $12 \mathrm{nT}$ at the sub-flow point. The magnetic field strength is also increased above and below the poles as the field drapes around Titan. The results for the other to cases in this plane are nearly identical.

The strange discontinuity in the electric field upstream of Titan requires further investigation. A three-dimensional isosurface of the $5 \times 10^{-4} \mathrm{~V} / \mathrm{m}$ level of the electric field strength from case 1 is shown in Fig. 21. The isosurface runs along the magnetic field and drapes over Titan above and below the poles. There is a twist in the isosurface upstream of Titan. This suggests that a corresponding kink should be present in the magnetic field from the curl of $\mathbf{E}$.

A detailed look at the components of the electric field in the orbital plane for case 1 is shown in Fig. 22. The component of $\mathbf{E}$ along the flow direction is shown in panel (A). There is a $3 \times 10^{-4} \mathrm{~V} / \mathrm{m}$ component pointing upstream (away from Titan) mainly in the anti-Saturn half of the simulation. While in the Saturn facing half of the simulation the electric field has a component pointing towards Titan with a magnitude of $8 \times 10^{-4} \mathrm{~V} / \mathrm{m}$. As a reminder the unperturbed convection electric field has a value of $6 \times 10^{-4}$ $\mathrm{V} / \mathrm{m}$ pointing away from Saturn. The magnitude of the electric field along the Saturn-Titan line is shown in panel (B). The largest value in this component points towards Titan and is located at the Saturn facing hemisphere. Could the build up in the electric field in this hemisphere is caused by the $\nabla P_{e}$ term? Panel (C) contains the results for the component of the electric field perpendicular to the orbital plane. Most of the buildup in this component is associated with the pickup ions. There is a small decrease in the field on the Saturn facing hemisphere $\left(\sim 1 \times 10^{-4} \mathrm{~V} / \mathrm{m}\right)$ but overall it is small compared to the other components of the electric field in this region. What determines the electric field configuration around Titan is an important issue. The two possible terms are the Hall term given by $\mathbf{J}_{e} \times \mathbf{B}$ or the $\nabla P_{e}$ term.

To test the effects of the Hall term the electron current density $\mathbf{J}_{e}$ was replaced by the ion current density $\mathbf{J}_{i}$ in Eq. (6). Contours of the resulting electric field magnitude 

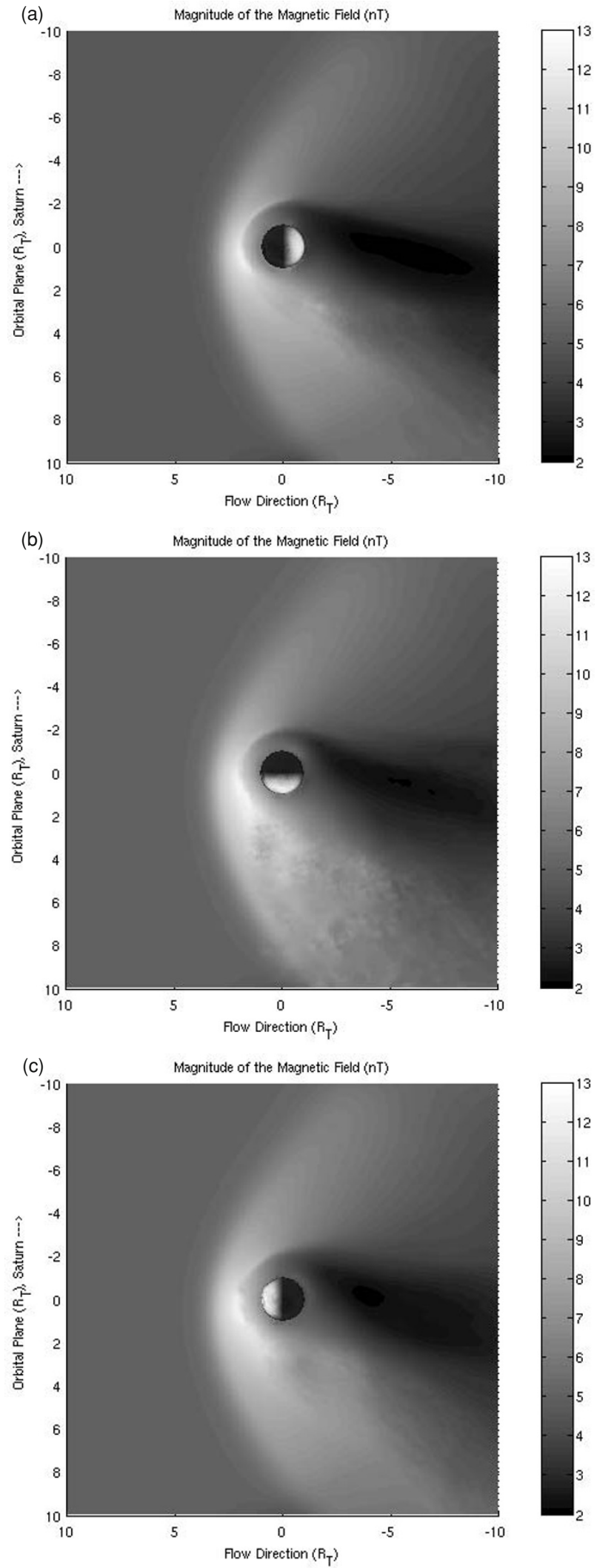

Fig. 26. The orbital view of the magnitude of the magnetic field for case 1 (A), case 2 (B) and case 3 (C) are shown.

are shown in Fig. 23. The electric field is now much more symmetric about the flow axis. As expected because the diamagnetic effects of the electric field are removed by the electrons with the ion flow. Thus the asymmetry in
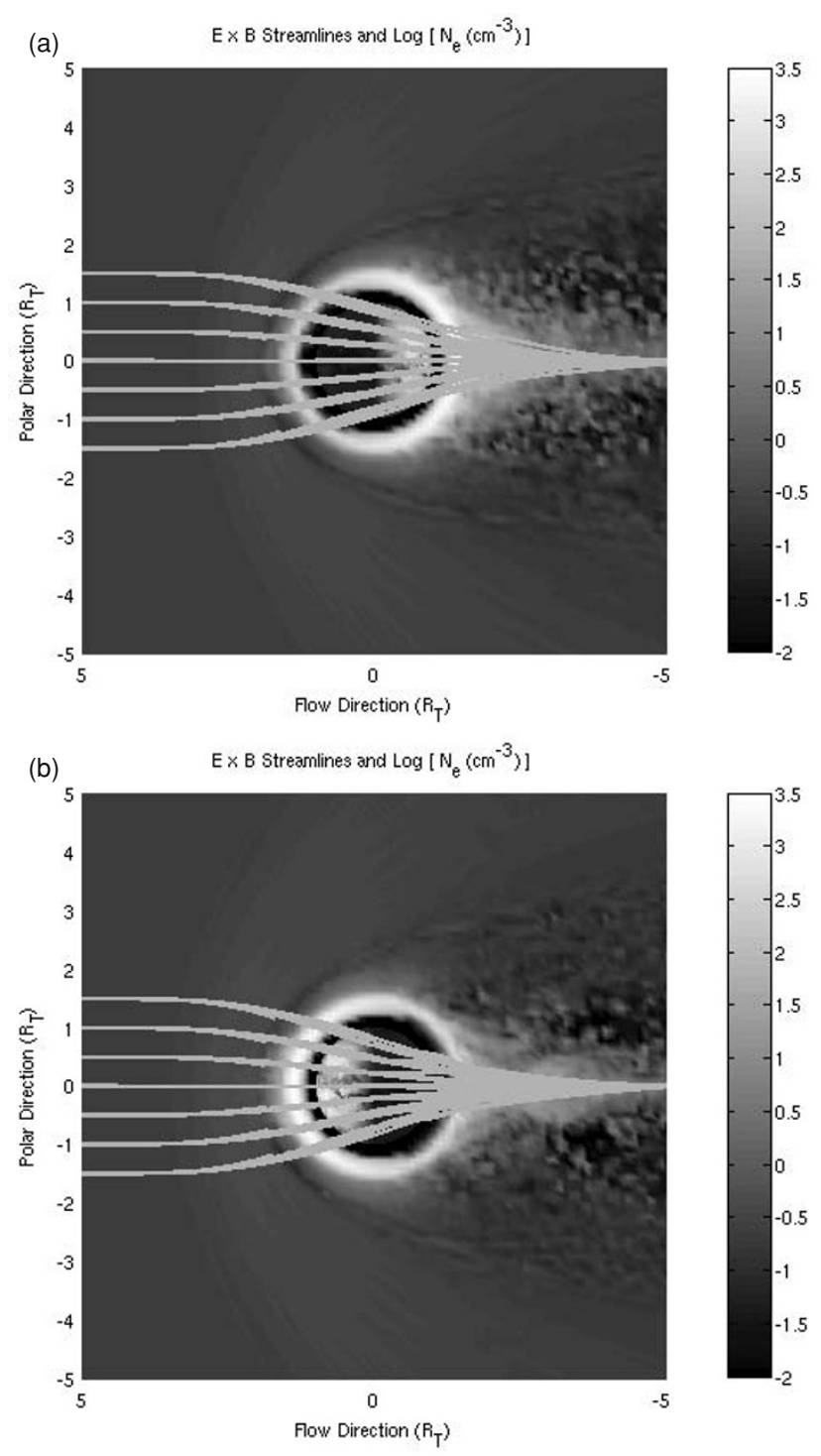

Fig. 27. Streamlines of $\mathbf{E} \times \mathbf{B}$ drift around Titan with contours of $\log \left(n_{e}\right)$ are shown for case 1 (A) and case 3 (B). The density follows the streamlines focusing in the wake. The streamlines are similar in each case.

the electric field was caused by the Hall term, or more correctly the electron currents and is a diamagnetic effect. However, there is still a small discontinuity in the electric field upstream of Titan.

The effects the $\nabla P_{e}$ term were also tested by removing it from Eq. (6). The resulting electric field magnitude in the orbital plane is shown in Fig. 24. Removing the $\nabla P_{e}$ term closed up the discontinuity in the electric field upstream of Titan. The $\nabla P_{e}$ term acts to reduce the electric field in the anti-Saturn facing hemisphere. Removal of the $\nabla P_{e}$ term did not remove the build up in the electric field in the Saturn facing hemisphere. The $\nabla P_{e}$ term is important and responsible partly for the structure of the electric field upstream of Titan.

Contours of the magnetic field in the polar view for case 1 are shown in Fig. 25. There is a build up in the magnetic field across the bow wave. The strongest build up occurs upstream of Titan and wraps around the poles as the field drapes around. The results for the other cases are identi- 


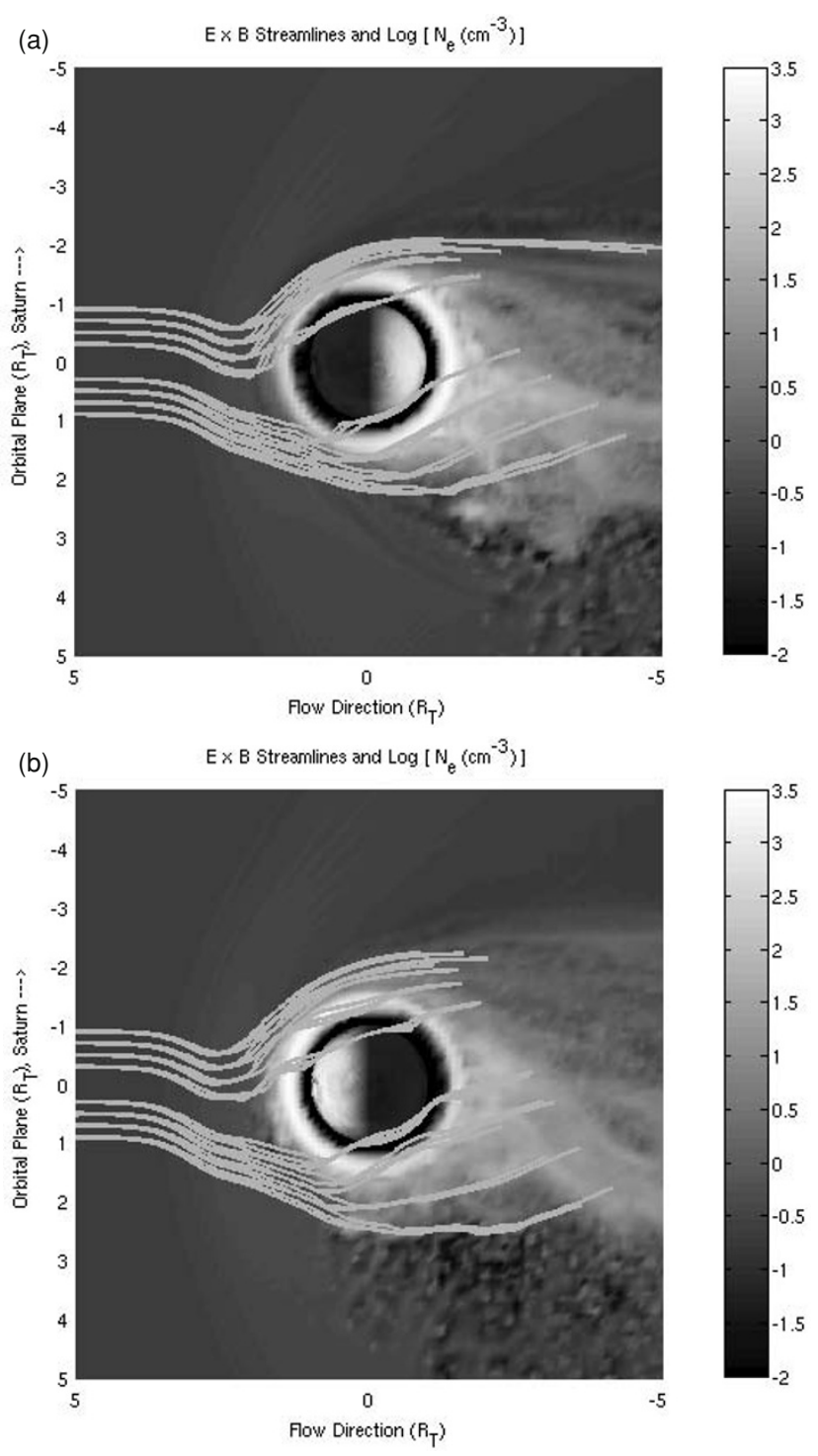

Fig. 28. Streamlines of $\mathbf{E} \times \mathbf{B}$ drift around Titan with contours of $\log \left(n_{e}\right)$ are shown for case 1 (A) and case 3 (B) in the orbital plane. Titan acts like astigmatic lens. The streamlines are similar in each case.

cal and are not shown. Figure 26 shows the magnetic field strength in the orbital plane. The field is asymmetric about the flow axis. The overall features are the same for each case. The magnetic field around Titan is not greatly influenced by the orientation of the dayside ionosphere.

Figure 27 shows stream lines of $\mathbf{E} \times \mathbf{B}$ overlaying the electron density contours in the polar view for cases 1 and 3. The streamlines essentially show the direction of flow of the electromagnetic energy given by the Poynting vector, $\mathbf{S}=c / 4 \pi \mathbf{E} \times \mathbf{B}$. Titan acts like a lens in this plane focusing the wave energy into the wake region. The contours show the plasma density also converging in the wake closely follow the streamlines. Figure 28 shows the orbital view for these two cases. Titan now acts like astigmatic lens. Streamlines that move along the Saturn facing hemisphere converge to the edge of the wake region. While streamlines that move along the anti-Saturn hemisphere diverge to fill in the wake. The streamlines shown are $3 \mathrm{di}-$ mensional where they appear to truncate is where they cross into the orbital plane. Streamlines that move along the antiSaturn facing hemisphere cross the orbital plane where the plasma is densest. There is a correlation between where the $\mathbf{E} \times \mathbf{B}$ streamlines converge and where the plasma density collects.

\section{Discussion}

The main conclusion that can be made from the results presented above is that the orientation of the dayside ionosphere with respect to the incident flow to first order does not have an effect on the global features of Titan's plasma interaction.

The ion loss rates are very close for each case. The radial flux of the ions passing through a spherical sampling region at $2500 \mathrm{~km}$ altitude show strong similarities in the location and magnitude of the ion fluxes. The reason for the similarities in the fluxes comes down to the configurations of the electric and magnetic fields. To first order the electric and magnetic fields around Titan are the same regardless of orientation of the dayside ionosphere. The resulting Poynting vector field has the same three-dimensional configuration as shown by the $\mathbf{E} \times \mathbf{B}$ streamlines in Figs. 27 and 28. The streamlines flow through the same regions in each case so the electromagnetic flux must go through the same regions. The ions respond to the electromagnetic flux and therefore the ion escape flux is nearly identical in all cases. The angle of illumination does not have a large effect on the ion loss from Titan.

There is a correlation between the relative amounts of each species being lost and the altitude of the ionospheric peak for that species. The higher the altitude of the ionospheric peak the larger the loss rate for that species. The main ion species lost by Titan are $\mathrm{M}^{+}$and $\mathrm{H}^{+}$. These correspond to the species $\mathrm{CH}_{5}{ }^{+}, \mathrm{N}^{+}, \mathrm{CH}_{4}{ }^{+}, \mathrm{CH}_{3}{ }^{+}, \mathrm{CH}_{2}{ }^{+}$, $\mathrm{CH}^{+}, \mathrm{C}^{+}$represented by $\mathrm{M}^{+}$in our model and $\mathrm{C}_{2} \mathrm{H}_{5}{ }^{+}$represented by $\mathrm{H}^{+}$. These species peak relatively high up in the ionosphere. The exception to the altitude trend is the loss of rate of the $\mathrm{L}^{+}$species.

The $\mathrm{L}^{+}$species peaks at the top of the ionosphere in the 1-dimensional photochemical model shown in Fig. 3. However, the peak ionization rate for this species occurs at an altitude of $1000 \mathrm{~km}$ (see Appendix A). The $\mathrm{L}^{+}$created below $1500 \mathrm{~km}$ is chemically used to create $\mathrm{M}^{+}$and $\mathrm{H}^{+}$. Above $1500 \mathrm{~km}$ the reactions that create these species shut off and the $\mathrm{L}^{+}$is allowed to build up in the photochemical model. The ionization rate is very small though less than $0.001 \mathrm{~cm}^{-3}$. Whatever $\mathrm{L}^{+}$is created above $1500 \mathrm{~km}$ gets picked up by Saturn's magnetosphere before it can build up and not much is being produced. The advection time scales and the chemical loss reaction rates are faster than the ionization rates for the $\mathrm{L}^{+}$species.

The net loss rates reported here do not show a strong dependence on the orientation of the dayside ionosphere. Using Cassini observations Wahlund et al. (2005) report a net ion loss rate of $1 \times 10^{25} \mathrm{~s}^{-1}$. Later estimates by Modolo et al. (2007) have upped the net loss rates to $2-7 \times 10^{25}$ $\mathrm{s}^{-1}$. The net loss rate reported here is about half the rate of Wahlund et al. Part of this discrepancy may be due to the recent announcement that the initial INMS neutral densities reported were a factor of 3 too low (Waite, private commu- 
nication). Increasing the neutral $\mathrm{CH}_{4}$ and $\mathrm{N}_{2}$ densities and the corresponding photo and secondary electron ionization rates will bring our ion loss rates into line with the results of Wahlund et al. and the lower limit reported by Modolo et al. There would be a 3 fold increase in the production of $\mathrm{M}^{+}$and $\mathrm{H}^{+}$by ionization of the $\mathrm{CH}_{4}$ and $\mathrm{N}_{2}$. The increase in the $\mathrm{M}^{+}$and $\mathrm{H}^{+}$densities will lead to an increase in the $\mathrm{H}^{+}, \mathrm{MHC}^{+}$and $\mathrm{HNI}^{+}$via several chemical reactions. A further increase in the loss rate could occur if the electron impact ionization rates were increased. The electron impact rates used in this study are based on the CAPS electron fluxes measured during the TA encounter. The electron spectrum during this encounter is classified by Rymer et al. (2009) as a plasma sheet encounter. The peak electron energies in this region run between $120-600 \mathrm{eV}$ with the fluxes at the peak energy between $3.5 \times 10^{5}$ and $1.2 \times 10^{6}$ $\mathrm{cm}^{-2} \mathrm{~s}^{-1} \mathrm{sr}^{-1}$ Rymer et al. (2009). Using electron production rates based on the higher end of these ranges would increase the nightside ionospheric densities and hence the ion loss from that region. There may be other factors that are not currently included in the present simulations that could contribute to ion loss from Titan. Neutral winds could help initially accelerate pickup ions leading potentially leading to a larger escape flux. Adding a realistic distribution of massive negative ions will change the currents in Titan's ionosphere and could also potentially add to a larger escape flux. Of course further refinements to the ion-neutral chemistry model could also contribute.

The simulation results show that below $1200 \mathrm{~km}$ altitude densities in Titan's ionosphere is in photochemical equilibrium. Above $1300 \mathrm{~km}$ altitude the densities are determined by the plasma advection. The plasma dynamics is of course determined by the electric and magnetic fields. The transition between chemical equilibrium and advection occurs somewhere between $1200-1300 \mathrm{~km}$. This is in agreement with the range predicted by Ma et al. (2006).

Around Titan both the Hall and $\nabla P_{e}$ have significant effects on the electric and resulting magnetic fields. The Hall term is responsible for the large scale asymmetry about the flow axis in the orbital plane. It does not have an effect on the fields in the polar plane. Near Titan, the $\nabla P_{e}$ is a major contributor to the electric field. It is responsible for the twist and discontinuity in the electric field present upstream of Titan's nose. It acts to decrease the electric field along the anti-Saturn hemisphere of Titan. The magnitude of the $\nabla P_{e}$ term is dependent on the electron density gradients in Titan's ionosphere and the electron temperature. The electron density gradients in our model are determined by the chemistry and ion transport. The electron temperature is treated adiabatically above $1400 \mathrm{~km}$ altitude. Below $1400 \mathrm{~km}$ the temperature is set and held constant to match the Langmuir probe results reported by Åren et al. (2009). This is reasonable since we do not include the electron-neutral cooling processes in the model. It is the region above our mask where the $\nabla P_{e}$ term is strongest.

To first order the electric and magnetic fields are in agreement between each of the cases. The higher order differences are due to ion pick up. The ion pick up, leads to blobs of plasma in the anti-Saturn facing flank. These plasma blobs feedback on the electric field creating waves and tur- bulence. This should be seen by Cassini out beyond $10 R_{\mathrm{T}}$ from Titan when approaching from beyond Titan's orbit. The turbulence does show a second order dependence on the location of the dayside ionosphere.

The main conclusions of this study, that the orientation of the dayside ionosphere has little effect on the ion loss rates from Titan is in agreement with the results of Sillanpää et al. (2006). There is a major topological difference between the present results and theirs. The ion tail in Sillanpää et al. was deflected by $45^{\circ}$ with respect to the incident flow. This deflection was in the direction towards Saturn and into the convection electric field. The deflection is in the opposite direction indicated by the testparticle studies of Luhmann (1996), Ledvina et al. (2000, 2004b, 2005) and Tseng et al. (2008). Sillanpää et al. attribute this deflection to a conservation of momentum. As ions are picked up from Titan they move in the direction of the convection electric field, away from Saturn. The incident flow then is deflected in the opposite direction (towards Saturn) to conserve momentum. The deflection of the incident flow leads to the slow moving pickup ions being pulled along towards Saturn. The results presented here (see Fig. 13) do not show a deflection of the ion tail towards Saturn. The reason for the differences in the simulation results must be due to how the ionospheres are handled in the simulations. The ionosphere in Sillanpää et al. was treated has a perfectly conducting sphere that uniformly emitted plasma. Thus the ionosphere was a solid object. The ionosphere in this study was much softer. Fields were allowed to penetrate it due to collisional resistivity. The collisions also dampened the ion motion. The ionospheric density was a result of the driving equations responding to the incident conditions. Additionally the $\nabla P_{e}$ was shown to have a important effect on the electric fields around Titan. This resulted in an obstacle that is not spherically symmetric, not a uniform emitter of plasma and not a perfectly conducting sphere. As a result the large deflection of the ion tail towards Saturn reported by Sillanpää et al. is not present in the current simulation results.

\section{Conclusions}

The main conclusion to be remembered via these simulations is that to first order the orientation of the dayside ionosphere with respect to the incident flow does not affect the global scale features of Titan's interaction with Saturn's magnetosphere.

The ionosphere is in photochemical equilibrium below $1200 \mathrm{~km}$. The transition between photochemical equilibrium and density profiles determined by advection occurs in a narrow shell $1200-1300 \mathrm{~km}$ altitude. The major ion species lost by Titan are represented by $\mathrm{M}^{+}$and $\mathrm{H}^{+}$, these correspond to $\mathrm{CH}_{5}{ }^{+}, \mathrm{N}^{+}, \mathrm{CH}_{4}{ }^{+}, \mathrm{CH}_{3}{ }^{+}, \mathrm{CH}_{2}{ }^{+}, \mathrm{CH}^{+}, \mathrm{C}^{+}$ and $\mathrm{C}_{2} \mathrm{H}_{5}{ }^{+}$. There is a correlation between the ion loss rate of a given species and the altitude of its peak ionospheric density. The lower the altitude of the ionospheric density peak the lower the loss rate. These results are not strongly dependent on the orientation of the dayside ionosphere.

The electric field at the sub-flow point upstream of Titan contains a twist. This twist should correspond to a twist or kink in the magnetic field lines in this region. The twist is caused by a combination of the Hall and $\nabla P_{e}$ terms. The 
Hall term is responsible for the asymmetries in the fields seen in the orbital plane around Titan. While the $\nabla P_{e}$ term acts to decrease the electric field in the anti-Saturn facing flank.

Titan acts as a lens in the polar plane focusing the electromagnetic wave energy given by the Poynting field into the wake. While it acts as an astigmatic lens in the orbital plane. The wave energy is converged in the Saturn facing hemisphere and diverged in the anti-Saturn facing hemisphere. The slow moving plasma follow Poynting field as reflected in the density.

Titan's interaction with Saturn's magnetosphere is a complex and multifaceted problem. The research presented here shows that what was thought to be a major issue, the orientation of the dayside ionosphere, only has second order effects on the global scale of the interaction. Other parameter variations in the incident plasma or field parameters, as would be the case at different locations in Saturn's magnetosphere should produce larger effects. While the second order effects are not important to the global interaction they may have a significant effect on the local evolution and energy balance of Titan's ionosphere, atmosphere and the interaction between them. Localized heating of the atmosphere by ion precipitation may have a significant impact on the neutral density profiles. In addition it has been suggested that the neutral winds may have an influence on how the magnetic field slides around Titan (Cravens et al., 2010). These are just some of the outstanding issues that remain to be addressed.

Acknowledgments. The authors of this paper would like to acknowledge the support from NASA grant NNH09CE73C, and NASA grant NNX08AK95G. In addition, the authors would like to acknowledge the computational support provided by the NASA Advanced Supercomputer, NAS, scientific computing facility at NASA Ames Research Center Moffett field CA.

\section{Appendix A.}

We group similar mass ions into seven generic ion species. These generic species, their mass and the real ion species they represent are shown in Table 1. The chemical reaction rates used were created by combining the relevant reactions from the updated model of Cravens et al. (2004). The photochemical equilibrium results shown Fig. 3 use the electron temperature profile calculated by Gan et al. (1992) for determining temperature dependent reaction rates. We include 11 neutral atmospheric species: $\mathrm{H}, \mathrm{H}_{2}, \mathrm{CH}_{4}, \mathrm{HCN}$, $\mathrm{N}_{2}, \mathrm{C}_{2} \mathrm{H}_{2}, \mathrm{C}_{2} \mathrm{H}_{4}, \mathrm{C}_{2} \mathrm{H}_{6}, \mathrm{C}_{3} \mathrm{H}_{4}, \mathrm{C}_{4} \mathrm{H}_{2}$ and $\mathrm{HC}_{3} \mathrm{~N}$. The altitude profiles for each species come from the model of Toublanc et al. (1995) with the exception of $\mathrm{N}_{2}$ and $\mathrm{CH}_{4}$ which come from the INMS profiles measured by Cassini during the Ta encounter reported by Waite et al. (2005). The density profiles for each of the neutral species used here are shown in Fig. A.1.

The results shown in this section do not include any dynamical processes such as ion transport. Hence we solve the following for each ion species:

$$
\frac{d n_{s}}{d t}=R_{s}-L_{s}
$$

Where $n_{s}$ is the number density of species $s, R_{S}$ is the net

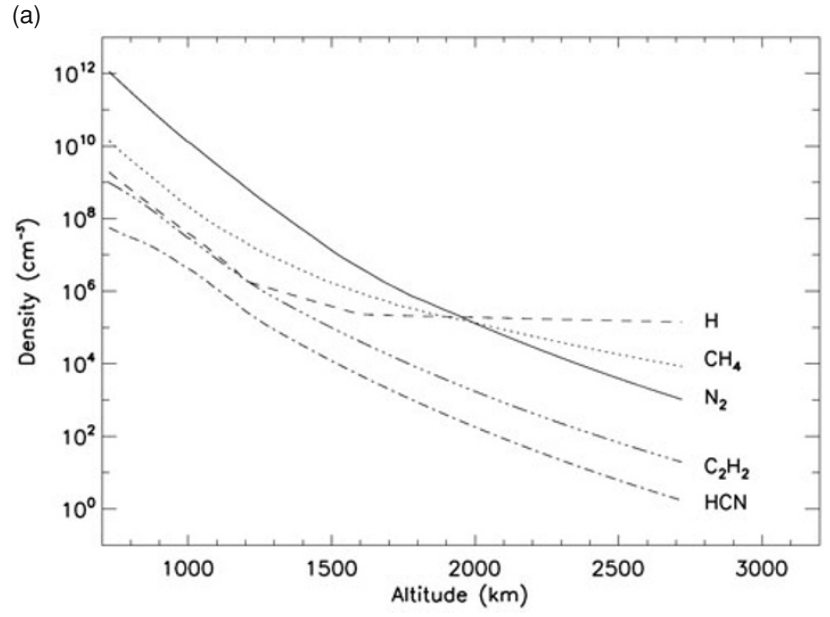

(b)

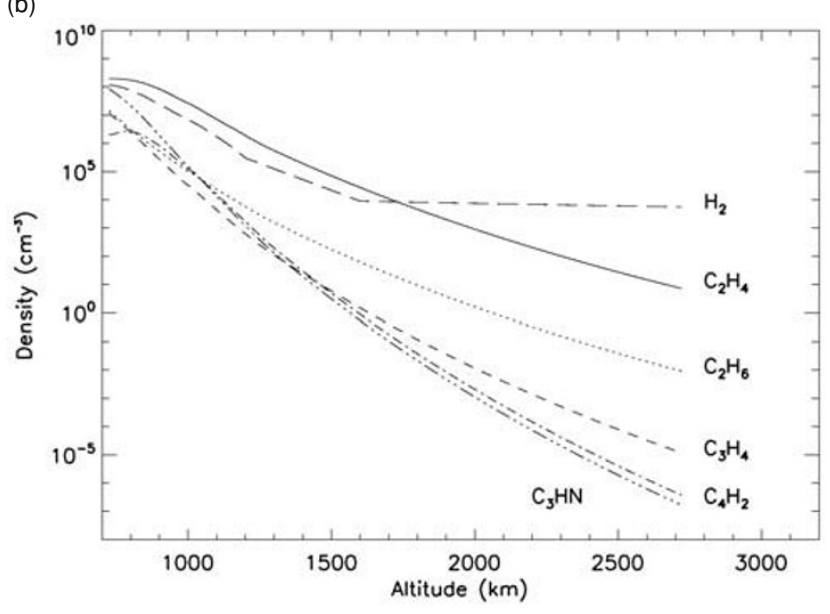

Fig. A.1. The neutral density profiles used in the ion-neutral chemical and the ion-neutral collision models.

production rate (photo and secondary electron impact ionization or superthermal electron impact ionization) plus the chemical production rate) and $L_{s}$ is the chemical loss rate. The primary production rates versus solar zenith angle (sza) are shown in Figs. A.2-A.4 for species $\mathrm{L}^{+}, \mathrm{M}^{+}$and $\mathrm{H}^{+}$ respectively. Production of these species drives the production of the remaining species. The ion production rates were calculated with a solar minimum solar flux and the above mentioned neutral atmosphere (cf. Cravens et al., 2005 for further details). There is substantial ion production beyond the terminator for each species. Also the ion production due to superthermal electron impact is comparable to photoionization at larger solar zenith angles (sza). The electron production rates are calculated using the two-stream method of Gan et al. (1993). The electrons are taken to be Maxwellian with a density of $0.1 \mathrm{~cm}^{-3}$ and a temperature of $100 \mathrm{eV}$ and move along a parabolic magnetic field line. This is consistent with the CAPS electron measurements during the T5 encounter and reported by Ågren et al. (2007). The ionization rates of $\mathrm{L}^{+}, \mathrm{M}^{+}$and $\mathrm{H} 1^{+}$due to super thermal electron impacts are shown in Fig. A.5.

Production of the $\mathrm{L}^{+}$ion is due to ionization of the light neutrals $\mathrm{H}$ and $\mathrm{H}_{2}$ (see Fig. A.2). No chemical production is assumed for the light species but chemical losses occur due 


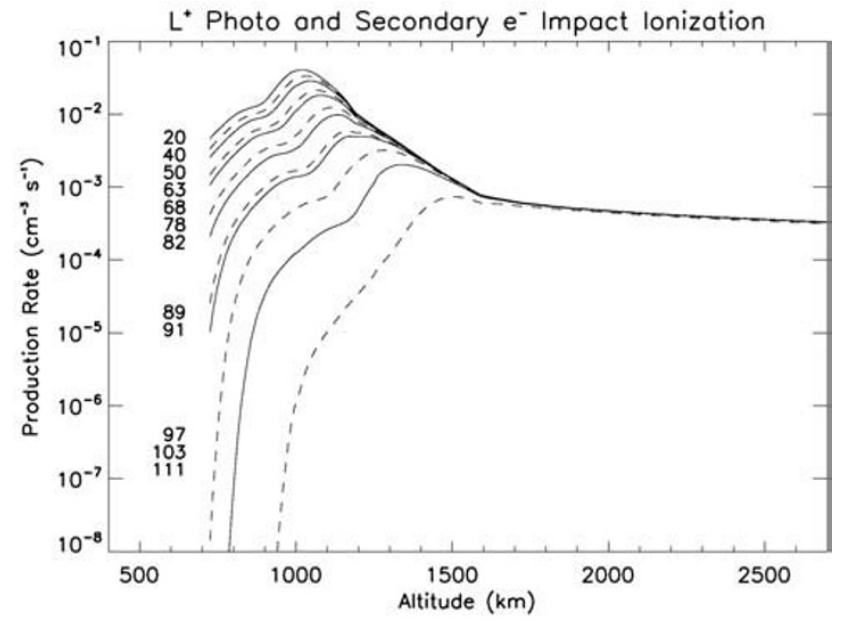

Fig. A.2. The ionization rates of the $\mathrm{L}^{+}$species versus altitude, due to photo and secondary $e^{-}$impact ionization are shown.

to ion-neutral reactions and dissociative recombination.

$$
\begin{array}{ll}
\left(\mathrm{H}, \mathrm{H}_{2}\right)+\gamma, e^{-} \rightarrow \mathrm{L}^{+} & K, \text { see Fig. A.2 } \\
\mathrm{L}^{+}+\mathrm{N}_{2} \rightarrow \mathrm{M}^{+}+\mathrm{X} & K=4.0 \times 10^{-10} \\
\mathrm{~L}^{+}+\mathrm{CH}_{4} \rightarrow \mathrm{H}^{+}+\mathrm{X} & K=1.3 \times 10^{-9} \\
\mathrm{~L}^{+}+e^{-} \rightarrow \mathrm{L} & \alpha=3.5 \times 10^{-12} \sqrt{300 / T_{e}}
\end{array}
$$

The $\mathrm{M}^{+}$species is produced by ionization of $\mathrm{N}_{2}$ and $\mathrm{CH}_{4}$ (see Fig. A.3). In addition $\mathrm{L}^{+}$can react with $\mathrm{N}_{2}$ to produce $\mathrm{M}^{+}$.

$$
\begin{array}{ll}
\left(\mathrm{N}_{2}, \mathrm{CH}_{4}\right)+\gamma, e^{-} \rightarrow \mathrm{M}^{+} & K, \text { see Fig. A.3 } \\
\mathrm{L}^{+}+\mathrm{N}_{2} \rightarrow \mathrm{M}^{+}+\mathrm{X} & K=4.0 \times 10^{-10} .
\end{array}
$$

The loss mechanisms for $\mathrm{M}^{+}$include recombination and interactions with neutrals to produce heavier species.

$$
\begin{array}{ll}
\mathrm{M}^{+}+e^{-} \rightarrow \mathrm{X} & \alpha=7.0 \times 10^{-7} \sqrt{300 / T_{e}} \\
\mathrm{M}^{+}+\mathrm{C}_{2} \mathrm{H}_{4} \rightarrow \mathrm{H}^{+}+\mathrm{X} & K=1.5 \times 10^{-9} \\
\mathrm{M}^{+}+\mathrm{C}_{2} \mathrm{H}_{6} \rightarrow \mathrm{H}^{+}+\mathrm{X} & K=2.0 \times 10^{-10} \\
\mathrm{M}^{+}+\mathrm{CH}_{4} \rightarrow \mathrm{H}^{+}+\mathrm{X} .8
\end{array}
$$

The resulting neutral species is represented by $\mathrm{X}$.

Production of the $\mathrm{H}^{+}$species is due to the ionization of $\mathrm{N}_{2}$ and $\mathrm{CH}_{4}$ (see Fig. A.4). Reactions of $\mathrm{L}^{+}$and $\mathrm{M}^{+}$with neutrals also contribute to the production of $\mathrm{H}^{+}$.

$$
\begin{array}{ll}
\left(\mathrm{N}_{2}, \mathrm{CH}_{4}\right)+\gamma, e^{-} \rightarrow \mathrm{H} 1^{+} & K \text {, see Fig. A.4 } \\
\mathrm{L}^{+}+\mathrm{CH}_{4} \rightarrow \mathrm{H}^{+}+\mathrm{X} & K=1.3 \times 10^{-9} \\
\mathrm{M}^{+}+\mathrm{C}_{2} \mathrm{H}_{4} \rightarrow \mathrm{H} 1^{+}+\mathrm{X} & K=1.5 \times 10^{-9} \\
\mathrm{M}^{+}+\mathrm{C}_{2} \mathrm{H}_{6} \rightarrow \mathrm{H} 1^{+}+\mathrm{X} & K=2.0 \times 10^{-10}
\end{array}
$$

Once produced the $\mathrm{H}^{+}$is lost by recombination and ion-

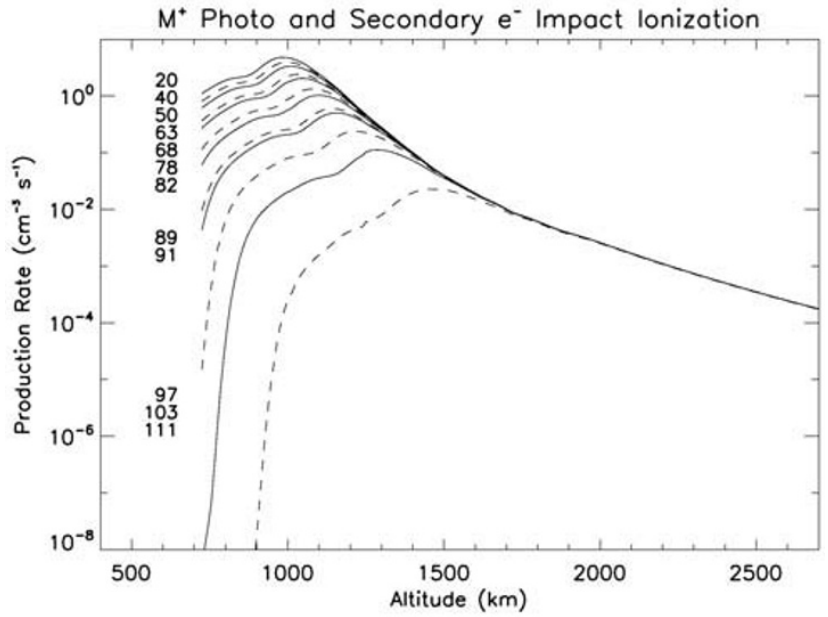

Fig. A.3. The ionization rates of the $\mathrm{M}^{+}$species versus altitude, due to photo and secondary $e^{-}$impact ionization are shown.

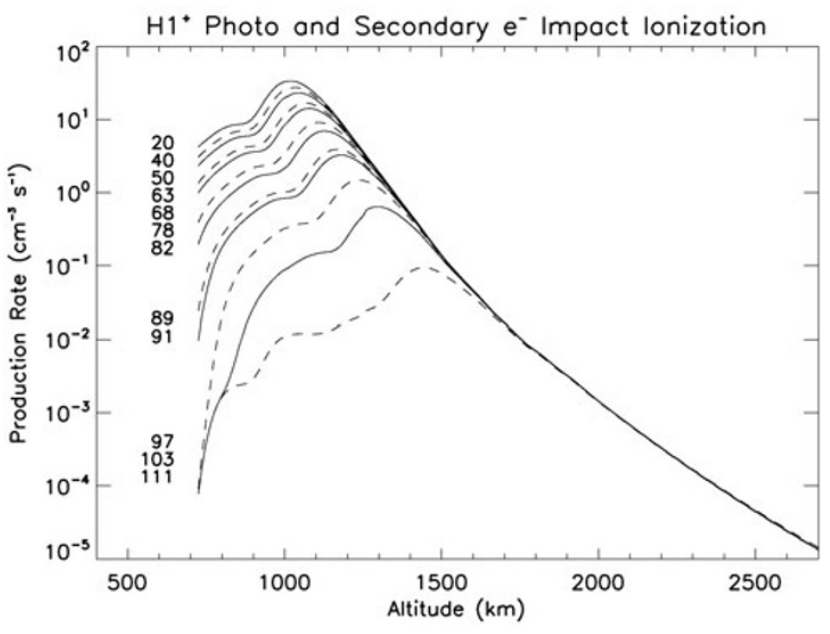

Fig. A.4. The ionization rates of the $\mathrm{H}^{+}$species versus altitude, due to photo and secondary $e^{-}$impact ionization are shown.

neutral reactions.

$$
\begin{array}{ll}
\mathrm{H}^{+}+e^{-} \rightarrow \mathrm{H} 1 & \\
\quad \alpha=1.9 \times 10^{-6} \sqrt{300 / T_{e}} & \\
\mathrm{H} 1^{+}+\mathrm{HCN} \rightarrow \mathrm{H}^{+}+\mathrm{X} & K=2.7 \times 10^{-9} \\
\mathrm{H}^{+}+\mathrm{C}_{2} \mathrm{H}_{2} \rightarrow \mathrm{MHC}^{+}+\mathrm{X} & K=3.9 \times 10^{-10} \\
\mathrm{H}^{+}+\mathrm{C}_{2} \mathrm{H}_{4} \rightarrow \mathrm{MHC}^{+}+\mathrm{X} & \\
\mathrm{H}^{+}+\mathrm{HC}_{3} \mathrm{~N} \rightarrow \mathrm{HNI}^{+}+\mathrm{X} & K=3.6 \times 10^{-9} .
\end{array}
$$

Each of the additional species in our model is produced by the interactions of ions with neutrals. For the $\mathrm{H}_{2}{ }^{+}$species the ions involved are $\mathrm{M}^{+}$and $\mathrm{H}^{+}$interacting with $\mathrm{CH}_{4}$ and $\mathrm{HCN}$ respectively.

$$
\begin{array}{ll}
\mathrm{M}^{+}+\mathrm{CH}_{4} \rightarrow \mathrm{H}^{+}+\mathrm{X} & K=1.0 \times 10^{-11} \\
\mathrm{H}^{+}+\mathrm{HCN} \rightarrow \mathrm{H}^{+}+\mathrm{X} & K=2.7 \times 10^{-9} .
\end{array}
$$


This species is lost through recombination and interactions with $\mathrm{C}_{4} \mathrm{H}_{2}$ and $\mathrm{HC}_{3} \mathrm{~N}$.

$$
\begin{aligned}
& \mathrm{H} 2^{+}+e^{-} \rightarrow \mathrm{H} 2 \\
& \quad \alpha=6.4 \times 10^{-7} \sqrt{300 / T_{e}} \\
& \mathrm{H}^{+}+\mathrm{C}_{4} \mathrm{H}_{2} \rightarrow \mathrm{MHC}^{+}+\mathrm{X}
\end{aligned}
$$

Reactions of $\mathrm{H}^{+}$and $\mathrm{H}_{2}^{+}$with neutrals create $\mathrm{MHC}^{+}$.

$$
\begin{array}{ll}
\mathrm{H}^{+}+\mathrm{C}_{2} \mathrm{H}_{2} \rightarrow \mathrm{MHC}^{+}+\mathrm{X} & K=1.0 \times 10^{-10} \\
\mathrm{H}^{+}+\mathrm{C}_{2} \mathrm{H}_{4} \rightarrow \mathrm{MHC}^{+}+\mathrm{X} & K=3.9 \times 10^{-10} \\
\mathrm{H} 2^{+}+\mathrm{C}_{4} \mathrm{H}_{2} \rightarrow \mathrm{MHC}^{+}+\mathrm{X} & K=1.6 \times 10^{-9} .
\end{array}
$$

This species is lost by recombination and reactions with neutral hydrocarbons.

$$
\begin{array}{rr}
\mathrm{MHC}^{+}+e^{-} \rightarrow \mathrm{MHC} & \\
\alpha=1.0 \times 10^{-6} \sqrt{300 / T_{e}} & \\
\mathrm{MHC}^{+}+\mathrm{C}_{2} \mathrm{H}_{2} \rightarrow \mathrm{HHC}^{+}+\mathrm{X} & K=4.0 \times 10^{-10} \\
\mathrm{MHC}^{+}+\mathrm{C}_{2} \mathrm{H}_{4} \rightarrow \mathrm{HHC}^{+}+\mathrm{X} & K=2.0 \times 10^{-10} \\
\mathrm{MHC}^{+}+\mathrm{C}_{3} \mathrm{H}_{4} \rightarrow \mathrm{HHC}^{+}+\mathrm{X} & K=6.0 \times 10^{-10} \\
& \\
\mathrm{MHC}^{+}+\mathrm{C}_{4} \mathrm{H}_{2} \rightarrow \mathrm{HHC}^{+}+\mathrm{X} & K=4.0 \times 10^{-10} .
\end{array}
$$

The species $\mathrm{HHC}^{+}$is created when $\mathrm{MHC}^{+}$interacts with hydrocarbons. The only loss process for this species is recombination.

$$
\begin{array}{lr}
\mathrm{MHC}^{+}+\mathrm{C}_{2} \mathrm{H}_{2} \rightarrow \mathrm{HHC}^{+}+\mathrm{X} & K=4.0 \times 10^{-10} \\
\mathrm{MHC}^{+}+\mathrm{C}_{2} \mathrm{H}_{4} \rightarrow \mathrm{HHC}^{+}+\mathrm{X} & K=2.0 \times 10^{-10} \\
\mathrm{MHC}^{+}+\mathrm{C}_{3} \mathrm{H}_{4} \rightarrow \mathrm{HHC}^{+}+\mathrm{X} & K=6.0 \times 10^{-10} \\
\mathrm{MHC}^{+}+\mathrm{C}_{4} \mathrm{H}_{2} \rightarrow \mathrm{HHC}^{+}+\mathrm{X} & K=4.0 \times 10^{-10}
\end{array}
$$

$$
\begin{aligned}
& \mathrm{HHC}^{+}+e \rightarrow \mathrm{HHC} \\
& \quad \alpha=1.0 \times 10^{-6} \sqrt{300 / T_{e}} .
\end{aligned}
$$

Our last ion species $\mathrm{HNI}^{+}$is created when $\mathrm{H}^{+}$and $\mathrm{H}_{2}^{+}$ interact with $\mathrm{HC}_{3} \mathrm{~N}$ and like $\mathrm{HHC}^{+}$it is only lost by recombination.

$$
\begin{array}{ll}
\mathrm{H}^{+}+\mathrm{HC}_{3} \mathrm{~N} \rightarrow \mathrm{HNI}^{+}+\mathrm{X} & K=3.6 \times 10^{-9} \\
\mathrm{H}^{+}+\mathrm{HC}_{3} \mathrm{~N} \rightarrow \mathrm{HNI}^{+}+\mathrm{X} & K=3.4 \times 10^{-9}
\end{array}
$$

$$
\begin{aligned}
& \mathrm{HNI}^{+}+e^{-} \rightarrow \mathrm{HNI} \\
& \alpha=1.0 \times 10^{-6} \sqrt{300 / T_{e}} .
\end{aligned}
$$

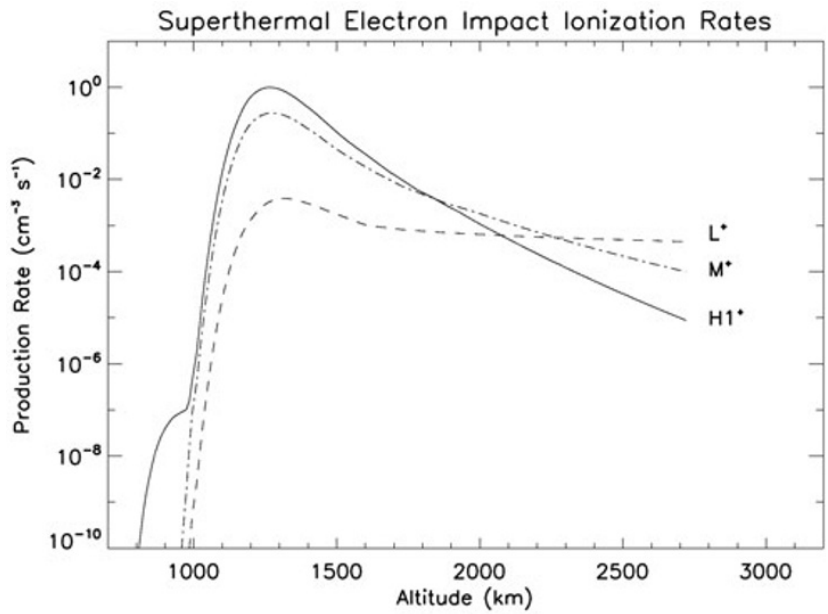

Fig. A.5. The ionization rates versus altitude due to super thermal electron impact ionization for each species are shown.

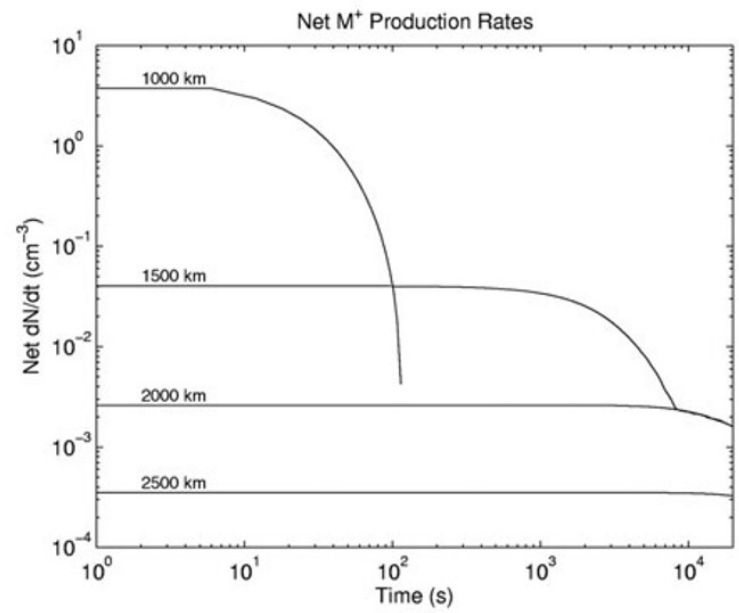

Fig. A.6. The net production rates for $\mathrm{M}^{+}$versus time for altitudes of $1000 \mathrm{~km}, 1500 \mathrm{~km}, 2000 \mathrm{~km}$ and $2500 \mathrm{~km}$ at a solar zenith angle of $0^{\circ}$.

Photoionization does not occur on the nightside. Instead the $\mathrm{L}^{+}, \mathrm{M}^{+}$and $\mathrm{H}^{+}$are created via electron impact ionization from superthermal electrons moving along magnetic field lines draped around Titan. We assume the simplest possible field configuration and take the field line to be radial with respect to Titan. The resulting ionization production rates are shown in Fig. A.5.

We solve the above ion-neutral chemistry network to obtain the altitude density profiles for each species. This is done using a simple explicit method. The time evolution of Titan's ionosphere as it responds to external influences in the hybrid simulations is the ultimate goal. It is not safe to assume that a steady equilibrium exists; therefore solving the chemistry implicitly is not valid. The ion-neutral chemistry must be solved explicitly. The explicit solution is sensitive to the chosen time step. It was found that if the selected time step was too large recombination of the $\mathrm{L}^{+}$would be over estimated. The result was the depletion of the $\mathrm{L}^{+}$and a reduction of $\mathrm{M}^{+}$and $\mathrm{H}^{+}$as well as their daughter products. A time step of $0.003 \mathrm{~s}$ was needed to accurately solve the ion-neutral chemistry. The final iono- 
spheric density profiles for both noon and midnight local time are shown in Fig. 3.

Figure A.6 shows the time evolution of the net $\mathrm{M}^{+}$production rate at 1000, 1500, 2000 and $2500 \mathrm{~km}$ altitudes. The production rate changes as the ion-neutral chemistry is solved and becomes zero when chemical equilibrium is reached. At $1000 \mathrm{~km}$ equilibrium is reach after only $100 \mathrm{~s}$. It takes about $10,000 \mathrm{~s}$ for the $\mathrm{M}^{+}$to come to equilibrium at $1500 \mathrm{~km}$. The $\mathrm{M}^{+}$takes longer to come to equilibrium than the simulation was run at the higher altitudes. It takes even longer for the heavier species that are derived from $\mathrm{M}^{+}$to come to equilibrium.

Figure A.6 implies that the simulations need to be started with a full ionosphere. It is not practical to run a hybrid simulation and build the ionosphere at the same time. Cassini INMS observations place Titan's exobase within an altitude range of 1400-1500 km. Previous work by Ma et al. (2006) found Titan's ionosphere is in chemical equilibrium at an altitude of $1200-1300 \mathrm{~km}$. This suggests that it would be safe to start the simulations with the ionosphere in chemical equilibrium at an altitude of $1500 \mathrm{~km}$. As the hybrid simulations are run the excess ionosphere will erode. It is not safe to assume that the ion-neutral chemistry will ever reach equilibrium while the ionosphere is being eroded. So the ion-neutral chemistry needs to be solved in a time accurate fashion. Figure A.6 also implies that it is not safe to use a constant ion production rate because the production rates are not constant.

\section{References}

Ågren, K. et al., On magnetospheric electron impact ionization and dynamics in Titan's ram-side and polar ionosphere - A Cassini case study, Ann. Geophys., 25, 2359-2369, 2007.

Ågren, K., J.-E. Wahlund, P. Garnier, R. Modolo, J. Cui, M. Galand, and I. Müller-Wodarg, On the ionospheric structure of Titan, Planet. Space Sci., 57, 1821-1827, doi:10.1016/j.pss.2009.04.012, 2009.

Ågren, K. et al., Detection of currents and associated electric fields in Titan's ionosphere from Cassini data, J. Geophys. Res., 116, A04313, doi:10.1029/2010JA016100, 2011.

Arridge, C. S., C. T. Russell, K. K. Khurana, N. Achilleos, N. Andre, A. M. Rymer, M. K. Dougherty, and A. J. Coates, Mass of Saturn's magnetodisc: Cassini observations, Geophys. Res. Lett., 34, L09108, doi:10.1029/2006GL028921, 2007.

Arridge, C. S., K. K. Khurana, C. T. Russell, D. J. Southwood, N. Achilleos, M. K. Dougherty, A. J. Coates, and H. K. Leinweber, Warping of Saturn's magnetospheric and magnetotail current sheets, J. Geophys. Res. (Space Physics), 113, A08217, doi:10.1029/2007JA012963, 2008.

Backes, H., F. M. Neubauer, M. K. Dougherty, H. Achilleos, N. Andre, C. S. Arridge, C. Bertucci, G. H. Jones, and K. K. Khurana, Titan's magnetic field signature during the first Cassini encounter, Science, 308, 992, 2005.

Bertucci, C. et al., The magnetic memory of Titan's ionized atmosphere, Science, 321(5895), 1475-1478, 2008.

Bertucci, C., B. Sinclair, N. Achilleos, P. Hunt, M. K. Dougherty, and C. S. Arridge, The variability of Titan's magnetic environment, Planet. Space Sci., 57, 1813, doi:10.1016/j.pss.2009.02.009, 2009.

Bird, M. K., R. Dutta-Roy, S. W. Asmar, and T. A. Rebold, Possible detection of Titan's ionosphere from Voyager 1 radio occultation observations, Icarus, 130, 426, 1997.

Brecht, S. H. and S. A. Ledvina, The solar wind interaction with the Martian Ionosphere/Atmosphere, Space Sci. Rev., 126, 15, 2006.

Brecht, S. H. and S. A. Ledvina, Control of ion loss from Mars during solar minimum, Earth Planets Space, 64, this issue, 165-178, 2012.

Brecht, S. H., J. G. Luhmann, and D. J. Larson, Simulation of the Saturnian magnetospheric interaction with Titan, J. Geophys. Res., 105, 13,11913,130, 2000.

Coates, A. J., F. J. Crary, G. R. Lewis, D. T. Young, J. H. Waite Jr., and
E. C. Sittler Jr., Discovery of heavy negative ions in Titan's ionosphere, Geophys. Res. Lett., 34, L22103, doi:10.1029/2007GL030978, 2007.

Coates, A. J., A. Wellbrock, G. R. Lewis, G. H. Jones, D. T. Young, F. J. Crary, and J. H. Waite Jr., Heavy negative ions in Titan's ionosphere: Altitude and latitude dependence, Planet. Space Sci., 57(14-15), 1866, 2009.

Crary, F. J., B. A. Magee, K. Mandt, J. H. Waite, J. Westlake, and D. T. Young, Heavy ions, temperatures and winds in Titan's ionosphere: Combined Cassini CAPS and INMS observations, Planet. Space Sci., 57, 1847-1856, 2009.

Cravens, T. E., C. J. Lindgren, and S. A. Ledvina, A two-dimensional multifluid MHD model of Titan's plasma environment, Planet. Space Sci., 46, 1193, 1998.

Cravens, T. C., J. Vann, J. Clark, J. Yu, C. N. Keller, and C. Brull, The ionosphere of Titan: an updated theoretical model, Adv. Space Res., 33, 212, doi:10.1016/ J.asr.2003.02.012, 2004.

Cravens, T. E. et al., Titan's ionosphere: model comparisons with Cassini Ta data, Geophys. Res. Lett., 32, L12108, doi:10.1029/2005GL023249, 2005.

Cravens, T. E. et al., Composition of Titan's ionosphere, Geophys. Res. Lett., 33, L07105, doi:10.1029/2005GL025575, 2006.

Cravens, T. E. et al., Model-data comparisons for Titan's nightside ionosphere, Icarus, 199, 174-188, 2009.

Cravens, T. E. et al., Dynamical and magnetic field time constants for Titan's ionosphere: Empirical estimates and comparisons with Venus, J. Geophys. Res., 115, A08319, doi:10.1029/2009JA015050, 2010.

Cui, J., M. Galand, R. V. Yelle, V. Vuitton, J. E. Wahlund, P. P. Lavvas, I. C. F. Muller-Wodarg, T. E. Cravens, W. T. Kasprzak, and J. H. Waite Jr., Diurnal variations of Titan's ionosphere, J. Geophys. Res., 114, A06310, doi:10.1029/2009JA014228, 2009.

Eviatar, A., G. L. Siscoe, J. D. Scudder Jr., E. C. Sittler, and J. D. Sullivan, The plumes of Titan, J. Geophys. Res., 87, 8091, 1982.

Fox, J. L. and R. V. Yelle, Hydrocarbon ions in the ionosphere of Titan, Geophys. Res. Lett., 24, 2179, 1997.

Galand, M., J. Lilensten, D. Toublanc, and S. Maurice, The ionosphere of Titan: ideal diurnal and nocturnal cases, Icarus, 140, 92, 1999.

Galand, M., R. Yelle, J. Cui, J.-E. Wahlund, V. Vuitton, A. Wellbrock, and A. Coates, Ionization sources in Titan's deep ionosphere, J. Geophys. Res., 115, A07312, doi:10.1029/2009JA015100, 2010.

Gan, L., C. N. Keller, and T. E. Cravens, Electrons in the ionosphere of Titan, J. Geophys. Res., 97, 12137, 1992.

Gan, L., T. E. Cravens, and C. N. Keller, A time-dependent model of suprathermal electrons at Titan, Plasma Environ. Non-Magnetic Planets, 4, 171, 1993 (proceedings of COSPAR colloquium held in Ann Arbor, MI, August 1992).

Garnier, P., J.-E. Wahlund, L. Rosenqvist, R. Modolo, K. Ågren, N. Sergis, P. Canu, M. Andre, D. A. Gurnett, W. S. Kurth, S. M. Krimigis, A. Coates, M. Dougherty, and J. H. Waite, Titan's ionosphere in the magnetosheath: Cassini RPWS results during the T32 flyby, Ann. Geophys., 27, 4257-4272, doi:10.5194/angeo-27-4257-2009, 2009.

Gurnett, D. A., F. L. Scarf, and W. S. Kurth, The structure of Titan's wake from plasma wave observations, J. Geophys. Res., 87, 1395, 1982.

Harned, D. S., J. Comput. Phys., 47, 452, 1982.

Hartle, R. E., E. C. Sittier, K. W. Ogilvie, J. D. Scudder, A. J. Lazarus, and S. K. Atreya, Titan's ion exosphere observed from Voyager1, J. Geophys. Res., 87, 1383, 1982.

Ip, W.-H., Titan's upper ionosphere, Astrophys. J., 362, 354, 1990.

Kabin, K., T. I. Gombosi, D. L. DeZeeuw, K. G. Powell, and P. L. Israelevich, Interaction of the Saturnian magnetosphere with Titan, J. Geophys. Res., 104, 2451, 1999.

Keller, C. N. and T. E. Cravens, One dimensional multispecies hydrodynamic models of the wakeside ionosphere of Titan, J. Geophys. Res., 99(A4), 6527-6536, 1994.

Keller, C. N., T. E. Cravens, and L. Gan, One-dimensional multispecies magnetohydrodynamic models of the ramside ionosphere of Titan, $J$. Geophys. Res., 99, 6511, 1994.

Keller, C. N., V. G. Anicich, and T. E. Cravens, Model of Titan's ionosphere with detailed hydrocarbon chemistry, Planet. Space Sci., 46, 1157-1174, 1998.

Kopp, A. and W.-H. Ip, Asymmetric mass loading effect at Titan's ionosphere, J. Geophys. Res., 106, 8323, 2001.

Ledvina, S. A. and T. E. Cravens, A three-dimensional MHD model of plasma flow around Titan, Planet. Space Sci., 46, 1175, 1998.

Ledvina, S. A., T. E. Cravens, A. Salman, and K. Kecskemety, Ion trajectories in Saturn's magnetosphere near Titan, Adv. Space Res., 26, 1691, 2000 . 
Ledvina, S. A., S. H. Brecht, and J. G. Luhmann, Ion distributions of 14 amu pickup ions associated with Titan's plasma interaction, Geophys. Res. Lett., 31, L17S10, doi:10.1029/2004GL019861, 2004a.

Ledvina, S. A., J. G. Luhmann, and T. E. Cravens, Ambient ion distributions in Saturn's magnetosphere near Titan during a non-Voyager type interaction, Adv. Space Res., 33, 221-226, 2004b.

Ledvina, S. A., T. E. Cravens, and K. Kecskemety, Ion distributions in Saturn's magnetosphere near Titan, J. Geophys. Res., 110, A06211, doi:10.1029/2004JA010771, 2005.

Ledvina, S. A., Y.-J. Ma, and E. Kallio, Modeling and simulating flowing plasmas and related phenomena, Space Sci. Rev., 139, 143-189, doi:10.1007/s11214-008-9384-6, 2008.

Luhmann, J. G., Titan's ion exospheric wake: A natural ion mass spectrometer?, J. Geophys. Res., 101, 29,387, 1996.

Ma, Y., A. F. Nagy, T. E. Cravens, I. G. Sokolov, J. Clark, and K. C. Hansen, 3-D global model prediction for the first close flyby of Titan by Cassini, Geophys. Res. Lett., 31, L22803, 2004.

Ma, Y.-J., A. F. Nagy, T. E. Cravens, I. U. Sokolov, K. C. Hansen, J.-E. Wahlund, F. J. Crary, A. J. Coates, and M. K. Dougherty, Comparisons between MHD model calculations and observations of Cassini flybys of Titan, J. Geophys. Res., 111, A05207, 2006.

Ma, Y.-J. and 10 colleagues, 3D global multispecies Hall-MHD simulation of the Cassini T9 flyby, Geophys. Res. Lett., 34(24), L24S10, 2007.

Mitchner, M. and C. H. Kruger, Jr., Partially Ionized Gases, John Wiley \& Sons, NY, USA, 1973. (a pdf is available online at: http://thermosciences.stanford.edu/research/part_ion_gas.html)

Modolo, R., G. M. Chanteur, J.-E. Wahlund, P. Canu, W. S. Kurth, D. Gurnett, A. P. Matthews, and C. Bertucci, Plasma environment in the wake of Titan from hybrid simulation: A case study, Geophys. Res. Lett., 34, L24S07, 2007.

Morooka, M. W., R. Modolo, J.-E. Wahlund, M. André, A. I. Eriksson, A. M. Persoon, D. A. Gurnett, W. S. Kurth, A. J. Coates, G. R. Lewis, K. K. Khurana, and M. Dougherty, The electron density of Saturn's magnetosphere, Ann. Geophys., 27, 2971-2991, doi:10.5194/angeo-272971-2009, 2009.

Müller, J., S. Simon, U. Motschmann, K. H. Glassmeier, J. Saur, J. Schüle, and G. J. Pringle, Magnetic field fossilization and tail reconfiguration in Titan's plasma environment during a magnetopause passage: 3D adaptive hybrid code simulations, Planet. Space Sci., doi:10.1016/j.pss.2010.07.018, 2010.

Nagy, A. F., Y. Liu, K. C. Hansen, K. Kabin, T. I. Gombosi, M. R. Combi, and D. L. DeZeeuw, The interaction between the magnetosphere of Saturn and Titan's ionosphere, J. Geophys. Res., 106, 6151, 2001.

Ness, N. F., M. H. Acuna, K. W. Behannon, and F. M. Neubauer, The induced magnetosphere of Titan, J. Geophys. Res., 87, 1369, 1982.

Persoon, A. M., D. A. Gurnett, W. S. Kurth, G. B. Hospodarsky, J. B. Groene, P. Canu, and M. K. Dougherty, Equatorial electron density measurements in Saturn's inner magnetosphere, Geophys. Res. Lett., 32, L23105, doi:10.1029/2005GL024294, 2005.

Persoon, A. M., D. A. Gurnett, W. S. Kurth, and J. B. Groene, A simple scale height model of the electron density in Saturn's plasma disk, Geophys. Res. Lett., 33, L18106, doi:10.1029/2006GL027090, 2006.

Rosenqvist, L., J. Wahlund, K. Agren, R. Modolo, H. J. Opgenoorth, D. Strobel, I. Muller-Wodarg, P. Garnier, and C. Bertucci, Titan ionospheric conductivities from Cassini measurements, Planet. Space Sci., 57, 1828-1833, doi:10.1016/j.pss.2009.01.007, 2009.
Rymer, A. M., H. T. Smith, A. Wellbrock, A. J. Coates, and D. T. Young, Discrete classification and electron energy spectra of Titan's varied magnetospheric environment, Geophys. Res. Lett., 36, L15109, doi:10.1029/2009GL039427, 2009.

Schunk, R. W. and A. F. Nagy, Ionospheres: Physics, plasma physics, and chemistry, in Cambridge Atmos. Space Sci. Ser., edited by Dressler, A. J., J. T. Houghton, and M. J. Rycroft, p. 104, Cambridge Univ. Press, Cambridge, U.K., 2000.

Sillanpää, I., E. Kallio, P. Janhunen, W. Schmidt, Mursula, J. Vilpolla, and P. Tanskanen, Hybrid simulation study of ion escape at Titan for different orbital positions, Adv. Space Res., 38, 799, 2006.

Simon, S., A. Boesswetter, T. Bagdonat, U. Motschmann, and K. H. Glassmeier, Plasma environment of Titan: A 3-d hybrid simulation study, Ann. Geophys., 24(3), 1113-1135, 2006.

Simon, S., A. Wennmachera, F. M. Neubauer, C. L. Bertucci, H. Kriegel, J. Saur, C. T. Russell, and M. K. Dougherty, Titan's highly dynamic magnetic environment: A systematic survey of Cassini magnetometer observations from flybys TA-T62, Planet. Space Sci., 58(10), 1230 1251, 2010a.

Simon, S., A. Wennmacher, F. M. Neubauer, C. L. Bertucci, H. Kriegel, C. T. Russell, and M. K. Dougherty, Dynamics of Saturn's magnetodisk near Titan's orbit: Comparison of Cassini magnetometer observations from real and virtual Titan flybys, Planet. Space Sci., 58, 1625-1635, doi:10.1016/j.pss.2010.08.006, 2010b.

Snowden, D., R. Winglee, C. Bertucci, and M. Dougherty, Threedimensional multifluid simulation of the plasma interaction at Titan, $J$. Geophys. Res., 112, A12221, doi:10.1029/2007JA012393, 2007.

Szego, K. et al., The global plasma environment of Titan as observed by Cassini Plasma Spectrometer during the first two close encounters with Titan, Geophys. Res. Lett., 32, L20S05, doi:10.1029/2005GL022646, 2005.

Toublanc, D., J. P. Parisot, J. Brillet, D. Gautier, F. Raulin, and C. P. McKay, Photochemical modeling of Titan's atmosphere, Icarus, 113, 2, 1995.

Tseng, W.-L., W.-H. Ip, and A. Kopp, Exospheric heating by pickup ions at Titan, Adv. Space Res., 42, 54, 2008.

Wahlund, J.-E. et al., Cassini measurements of cold plasma in the ionosphere of Titan, Science, 308, 986-989, 2005.

Wahlund, J.-E., M. Galand, I. Muller-Wodarg, J. Cui, R. V. Yelle, F. J. Crary, K. Mandt, B. Magee, J. H. Waite Jr., D. T. Young, A. J. Coates, P. Garnier, K. Agren, M. Andre, A. I. Eriksson, T. E. Cravens, V. Vuitton, D. A. Gurnett, and W. S. Kurth, On the amount of heavy molecular ions in Titan's ionosphere, Planet. Space Sci., 57, 14-15, 1857, 2009.

Waite, J. H., Jr., H. Niemann, R. V. Yelle, W. T. Kasprzak, T. E. Cravens, J. G. Luhmann, R. L. McNutt, W.-H. Ip, D. Gell, V. de La Haye, I. MullerWordag, B. Magee, N. Borggren, S. Ledvina, G. Fletcher, E. Walter, R. Miller, S. Scherer, R. Thorpe, J. Xu, B. Block, and K. Arnett, Ion neutral mass spectrometer results from the first flyby of Titan, Science, 308, 982-986, 2005.

Wolf, D. A. and F. M. Neubauer, Titan's highly variable plasma environment, J. Geophys. Res., 87, 881, 1982.

S. A. Ledvina (e-mail: ledvina@ssl.berkeley.edu), S. H. Brecht, and T. E. Cravens 